\title{
5
}

\section{Quantum Flows}

We develop a rather general framework for constructing quantum Markov processes through Markov operator cocycles (see Chapter 2, Section 3) that satisfy a quantum stochastic differential equation.

In order to achieve this goal we first recall the basic facts of Boson Fock quantum stochastic calculus and then give the fundamental results in the theory of quantum stochastic differential equations concerning existence, uniqueness, time reversal, isometricity and coisometricity of solutions. Next we construct the quantum flow associated with a Markov operator cocycle, and give a condition that guarantees that the restriction to a commutative subalgebra is a commutative flow.

As an application we show that every classical multidimensional diffusion process (with covariance and drift as in Chapter 4, Section 2) can be realised as a restriction to a commutative subalgebra of $\mathcal{B}(h)$ of a quantum flow.

Our processes are, in particular, Evans-Hudson flows [36].

\subsection{Quantum stochastic calculus}

In this section we give a short outline of the quantum stochastic calculus of Hudson and Parthasarathy [58] in a Boson Fock space which is the main tool for the construction of Markov operator cocycles. We refer to the monographs [68] and [74] for a complete exposition with proofs.

A quantum stochastic calculus can be developed in several frameworks: Fermion Fock space, Full Fock space, finite difference Fock space ...; we refer to [4] for a unified approach.

There exists several approaches also to quantum stochastic calculus in BosonFock space (see [13], [14], [58], [65], [66], [84] ...): we shall use here a notation introduced by Belavkin [16] as described by Parthasarathy (see, for example, [69], Sect. 2).

Let $h, k$ be two complex separable Hilbert spaces and let $\left\{e_{\ell} \mid \ell \in S\right\}$ be an orthonormal basis in $k$ ( $S$ is to be thought of as a subset of $I N$ ). Let $\mathcal{M}_{S}$ 
denote the dense linear submanifold of $L^{2}\left(\mathbb{R}_{+}\right) \otimes k$ of elements $f$ satisfying $\left\langle e_{\ell}, f(t)\right\rangle_{k}=0$ for all $t \geq 0$ for all but a finite number of indices $\ell$ and let $\mathcal{M}_{S}^{0}$ be the submanifold of continuous functions in $\mathcal{M}_{S}$. Let $\vec{S}=S \cup\{-\infty,+\infty\}$ and consider the Hilbert spaces

$$
\begin{gathered}
\hat{k}=\mathscr{C} e_{-\infty} \oplus k \oplus \mathscr{C} e_{+\infty}, \\
\mathcal{H}=h \otimes \Gamma\left(L^{2}\left(\mathbb{R}_{+}\right) \otimes k\right), \quad \widehat{\mathcal{H}}=h \otimes \hat{k} \otimes \Gamma\left(L^{2}\left(\mathbb{R}_{+}\right) \otimes k\right)
\end{gathered}
$$

where $\Gamma(\mathcal{K})$ denotes the Boson Fock space over a Hilbert space $\mathcal{K}$.

The uniform operator norm in $\mathcal{B}(\widehat{\mathcal{H}})$ will be denoted by $\|\cdot\|_{\infty}$. The exponential vector in the Boson Fock space $\Gamma(\mathcal{K})$ with test function $f \in \mathcal{K}$ will be denoted by $e(f)$ where

$$
e(f)=\sum_{n \geq 0} \frac{f^{\otimes n}}{\sqrt{n !}}
$$

For all $t>0$, we have the tensor product decomposition

$$
\mathcal{H}=h \otimes \Gamma\left(L^{2}(0, t) \otimes k\right) \otimes \Gamma\left(L^{2}(t, \infty) \otimes k\right) .
$$

Let $e\left(0_{t]}\right)$ and $e\left(0_{[t}\right)$ denote respectively the Fock vacuum in the Boson Fock spaces $\Gamma\left(L^{2}(0, t) \otimes k\right)$ and $\Gamma\left(L^{2}(t, \infty) \otimes k\right)$. Both $h \otimes \Gamma\left(L^{2}(0, t) \otimes k\right)$ and $h \otimes$ $\Gamma\left(L^{2}(t, \infty) \otimes k\right)$ can be identified with two subspaces of $\mathcal{H}$

$$
h \otimes \Gamma\left(L^{2}(0, t) \otimes k\right) \otimes e\left(0_{[t}\right), \quad h \otimes e\left(0_{t]}\right) \otimes \Gamma\left(L^{2}(t, \infty) \otimes k\right),
$$

via the unitary isomorphisms

$$
\begin{aligned}
u \otimes e\left(f 1_{(0, t)}\right) & \rightarrow u \otimes e\left(f 1_{(0, t)}\right) \otimes e\left(0_{[t}\right) \\
u \otimes e\left(f 1_{(t, \infty)}\right) & \rightarrow u \otimes e\left(f 1_{(t, \infty)}\right) \otimes e\left(0_{t]}\right) .
\end{aligned}
$$

Let us consider the ${ }^{*}$-algebra $\mathcal{B}=\mathcal{B}(\mathcal{H})$ of all bounded operators on $\mathcal{H}$. Let $\varphi_{0}$ be the state on the *-algebra of all bounded operators on $\Gamma\left(L^{2}\left(\mathbb{R}_{+}\right) \otimes k\right)$ associated with the vacuum vector $e(0)$

$$
\varphi_{0}: \mathcal{B}\left(\Gamma\left(L^{2}\left(\mathbb{R}_{+}\right) \otimes k\right)\right) \rightarrow \mathscr{C}, \quad \varphi_{0}(B)=\langle e(0), B e(0)\rangle .
$$

For any state $\rho$ on $\mathcal{B}(h)$ the map

$$
\varphi: \mathcal{B} \rightarrow \mathscr{C}, \quad \varphi=\rho \otimes \varphi_{0}
$$

is a state on $\mathcal{B}$. The pair $(\mathcal{B}, \varphi)$ is a quantum probability space.

Because of the embeddings (5.2), if we set

$$
\mathcal{B}_{0]}=\mathcal{B}(h), \quad \mathcal{B}_{t]}=\mathcal{B}\left(h \otimes \Gamma\left(L^{2}(0, t) \otimes k\right)\right)
$$

for all $t \geq 0$, then we obtain filtration of $\mathcal{B}$.

For all $t \geq 0$ we can define a conditional expectation

$$
\mathbb{E}_{t \mid}: \mathcal{B} \rightarrow \mathcal{B}_{t]}
$$


as follows. Let $B$ be an element of $\mathcal{B}$. The sesquilinear form on $h \otimes \Gamma\left(L^{2}(0, t) \otimes k\right)$

$$
(v, u) \rightarrow\left\langle\left(v \otimes e\left(0_{i t}\right)\right), B\left(u \otimes e\left(0_{[t}\right)\right)\right\rangle
$$

$\left(v \cdot u \in h \otimes \Gamma\left(L^{2}(0, t) \otimes k\right)\right)$ is bounded. The conditional expectation of $B$ with respect to $\mathcal{B}_{l]}$, denoted $\mathbb{E}_{t]}[B]$, is the element of $\mathcal{B}_{t]}$ representing this sesquilinear form. Denoting by $\Pi_{t]}$ the orthogonal projection of $\mathcal{H}$ onto the closed subspace $\left.h \otimes \Gamma\left(L^{2}(0, t) \otimes k\right)\right) \otimes e\left(0_{[t}\right)$ we have

$$
\mathbb{E}_{t]}[L]=\left.\Pi_{t\}} L \Pi_{\ell]}\right|_{h \Leftrightarrow \mathrm{r}\left(L^{2}(0, t) \otimes k\right)} \otimes \mathbb{1}_{\Gamma\left(L^{2}(t, \infty) \otimes k\right)} .
$$

The properties of conditional expectations (see Chapter 1, Section 4) are easily checked. Clearly, the family $\left(\mathbb{E}_{t]}\right)_{t \geq 0}$ is also projective.

For ach $t \geq 0$ let $\sigma_{t}$ be the right shift on the interval $[0, t]$ defined on $L^{2}\left(\mathbb{R}_{+}\right) \times k$ by

$$
\left(\sigma_{\ell} f\right)(x)=\left\{\begin{array}{cc}
f(x-t) & \text { if } x>t \\
0 & \text { if } x \leq t
\end{array} .\right.
$$

Let $\Gamma\left(\sigma_{t}\right)$ be the operators in $\Gamma\left(L^{2}\left(\mathbb{R}_{T}\right) \times k\right)$ defined by second quantization of $\sigma_{t}$,

$$
\Gamma\left(\sigma_{l}\right) c(f)=c\left(\sigma_{l} f\right)
$$

for all $f \in L^{2}\left(\mathbb{R}_{+}\right) \otimes k$. The operators $\sigma_{t}$ and $\Gamma\left(\sigma_{t}\right)$ are isometries for every $t \geq 0$. Notice that, for all $s, t \geq 0$ we have

$$
\Gamma\left(\sigma_{s}\right)^{*} \Gamma\left(\sigma_{t+s}\right)=\Gamma\left(\sigma_{t}\right), \quad \Gamma\left(\sigma_{s}\right) \Gamma\left(\sigma_{t}\right)=\Gamma\left(\sigma_{s+t}\right) .
$$

For each $s \geq 0$ and each bounded operator $x \in \mathcal{B}(\mathcal{H})$ the operator $\Gamma\left(\sigma_{s}\right) x \Gamma\left(\sigma_{s}\right)^{*}$ maps $h \otimes \Gamma\left(L^{2}(s, \infty) \otimes k\right)$ into itsclf. Indeed we have the diagram

$$
\begin{gathered}
\Gamma\left(\sigma_{s}\right)^{*} x \quad \Gamma\left(\sigma_{s}\right) \\
h \bowtie \Gamma\left(L^{2}(s, \infty) \otimes k\right) \longrightarrow \mathcal{H} \longrightarrow \mathcal{H} \longrightarrow h \otimes \Gamma\left(L^{2}(s, \infty) \otimes h\right)
\end{gathered}
$$

The canonical extension of $\Gamma\left(\sigma_{s}\right) x \Gamma\left(\sigma_{s}\right)^{*}$ to $\mathcal{H}$ via ampliation will be denoted by $\theta_{s}(x)$. Clearly $\left(\theta_{s}\right)_{s \geq 0}$ is a family of covariant shifts on $\mathcal{B}(\mathcal{H})$. Moreover, for all $x \in \mathcal{B}(h)$ and all $s \geq 0$, we have

$$
\theta_{s}(x)=x .
$$

This makes an important and relevant difference with the usual shift of classical Markov processes (see [2], [67]).

It is easy to see that $\theta$ is a covariant shift with respect to the family $\left(I E_{\ell]}\right)_{t \geq 0}$ of conditional expectations.

We now introduce the operator cocycles that we shall construct by means of quantum stochastic calculus.

Definition 5.1 A family $(X(t)\}_{0}$ of bounded operators in $\mathcal{H}$ is called a left cocycle (resp. right cocyclo) if for erery t. $n \geq 0$ we have

$$
X(t+s)=X(s) \theta_{s}(X(t)), \quad\left(\operatorname{resp} . X(+\cdots s) \cdots \theta_{s}(X(t)) X(s)\right)
$$


Let $F$ be the unique unitary operator in $\hat{k}$ defined by

$$
F e_{\ell}=e_{\ell} \quad \text { if } \quad \ell \in S, \quad F e_{+\infty}=e_{-\infty}: \quad F e_{-\infty}=e_{+\infty},
$$

and let $E_{-\infty}, E, E_{+\infty}$ be the orthogonal projections of $\hat{k}$ onto $\mathbb{C} e_{-\infty}, k, \mathbb{C} e_{+\infty}$ respectively. We denote by $\mathbb{B}$ the Belaukin algebra associated with $(h, k)$

$$
\mathbb{B}=\left\{L \in \mathcal{B}(h \otimes \hat{k}) \mid L u e_{-\infty}=L^{*} u e_{+\infty}=0 \text { for all } u \in h\right\}
$$

The map $L \mapsto L^{b}$ where $L^{b}=F L^{*} F$ is an involution of $\mathbb{B}$.

Let us introduce the quantum noises in $\widehat{\mathcal{H}},\left\{\Lambda_{m}^{\ell} \mid \ell, m \in \bar{S}\right\}$ defined by

$$
\begin{aligned}
\Lambda_{m}^{+\infty}(t) & =A^{+}\left(1_{(0, t)} \otimes\left|e_{m}\right\rangle\right), & & \text { if } m \in S, \ell=+\infty \\
\Lambda_{m}^{\ell}(t) & =\Lambda\left(1_{(0, t)} \otimes\left|e_{m}\right\rangle\left\langle e_{\ell}\right|\right), & & \text { if } m, \ell \in S \\
\Lambda_{-\infty}^{\ell}(t) & =A\left(1_{(0, t)} \otimes\left\langle e_{\ell}\right|\right), & & \text { if } \ell \in S, m=-\infty \\
\Lambda_{-\infty}^{+\infty}(t) & =t \mathbb{1} & & \text { if } \ell=+\infty, m=-\infty \\
\Lambda_{m}^{\ell}(t) & =0 & & \text { otherwise }
\end{aligned}
$$

where $A^{+}, A, \Lambda$ denote respectively the creation, amnihilation and gange operators in $\Gamma\left(L^{2}\left(\mathbb{R}_{+}\right) \bowtie k\right)$ defined, for each $u \in h$ and each exponential vector $e(f)$ by

$$
\begin{aligned}
A^{+}\left(1_{(0, t)} \otimes\left|e_{m}\right\rangle\right) u e(f) & =\left.\frac{d}{d \epsilon} u e\left(f+\epsilon 1_{(0, t)} e_{m}\right)\right|_{\epsilon=0} \\
\Lambda\left(1_{(0, t)} \otimes\left|e_{m}\right\rangle\left\langle e_{\ell}\right|\right) u e(f) & =-\left.i \frac{d}{d \epsilon} u e\left(e^{i \epsilon 1_{(0, t)} \otimes\left|e_{m}\right\rangle\left\langle e_{\ell}\right|} f\right)\right|_{\epsilon=0} \\
A\left(1_{(0, t)} \otimes\left\langle e_{\ell}\right|\right) u e(f) & =\left\langle e_{\ell} 1_{(0, t)}, f\right\rangle u e(f)
\end{aligned}
$$

where $\left|e_{m}\right\rangle\left\langle e_{\ell}\right|$ denotes the operator on $k$

$$
\left|e_{m}\right\rangle\left\langle e_{\ell}\right| x=\left\langle e_{\ell} \cdot x\right\rangle e_{m} .
$$

Notice that quantum noises $\Lambda_{m}^{\ell}$ with either $\ell \in S$ or $m \in S$ are martingales with respect to the family of conditional expectations $\left(\mathbb{E}_{t]}\right)_{t \geq 0}$.

Let $D$ be a dense linear submanifold of $h$ and let $D_{S}, \mathcal{D}_{S}$ and $\widehat{\mathcal{D}}_{S}$ be the dense linear submanifolds of $h \otimes k, \mathcal{H}$ and $\widehat{\mathcal{H}}$ gencrated by $\left\{u e_{\ell} \mid u \in D, \ell \in \bar{S}\right\}$, $\left\{u e(f) \mid u \in D, f \in \mathcal{M}_{S}\right\},\left\{u e_{e} e(f) \mid u \in D, \ell \in \bar{S}, f \in \mathcal{M}_{S}\right\}$ respectively.

Definition 5.2 A family $(L(t))_{t \geq 0}$ of opcrators in $\widehat{\mathcal{H}}$ is called $\left(D_{S}, \mathcal{M}_{S}\right)$-adapted if it satisfies the following conditions:

1. the domain of $L(t)$ contains $\widehat{\mathcal{D}}_{S}$ for all $t \geq 0$,

2. for each $\xi \in D_{S}, f \in \mathcal{M}_{S}, t \geq 0$ we have the tensor product factorisation

$$
L(t) \xi e(f)=\left\{L(t) \xi e\left(1_{(0, t)} f\right)\right\} \otimes e\left(1_{(t,+\infty)} f\right) .
$$


$A\left(D_{S}, \mathcal{M}_{S}\right)$-adapted family of operators $L$ is called $\left(D_{S}, \mathcal{M}_{S}\right)$-regular if the map

$$
t \mapsto L(t) \xi e(f)
$$

is continuous for all $\xi \in D_{S}, f \in \mathcal{M}_{S}$.

In a similar way one can define $\left(D, \mathcal{M}_{S}^{0}\right)$ and $\left(D, \mathcal{M}_{S}\right)$-adapted regular families of operators.

A $\left(D, \mathcal{M}_{S}\right)$-adapted family of operators $X=(X(t))_{t>0}$ in $\mathcal{H}$ is called unitary, isometric, coisometric, contractive if, for all $t \geq 0$, the operators $X(t)$ are unitary, isometric, coisometric, contractive.

Let $\mathcal{I}\left(D_{S}, \mathcal{M}_{S}\right)$ be the vector space of regular $\left(D_{S}, \mathcal{M}_{S}\right)$-adapted families of oprators $L$ in $\widehat{\mathcal{H}}$ satisfying

$$
\left\langle v e_{+\infty} e(g), L(t) u e_{\ell} e(f)\right\rangle=0, \quad L(t) u e_{-\infty} e(f)=0
$$

for all $u, v \in D, f, g \in \mathcal{M}_{S}, \ell \in \bar{S}$. With every $L \in \mathcal{I}\left(D_{S}, \mathcal{M}_{S}\right)$ it is possible to associate the set $\left\{L_{m}^{\ell} \mid \ell, m \in \bar{S}\right\}$ of $\left(D, \mathcal{M}_{S}\right)$-adaptcd regular families of operators in $\widehat{\mathcal{H}}$ defined by

$$
\left\langle v e(g), L_{m}^{\ell}(t) u e(f)\right\rangle=\left\langle v e_{\ell} e(g), L(t) u e_{m} e(f)\right\rangle .
$$

In the new notation one can write the stochastic integral

$$
\Lambda_{L}(t)=\int_{0}^{t} \sum_{\ell, m \in \bar{S}} L_{m}^{\ell}(t) d \Lambda_{\ell}^{m}(t)
$$

on the domain $\mathcal{D}_{S}$ as a regular $\left(D, \mathcal{M}_{S}\right)$-adapted family of operators. This is very convenient in the computations with the Ito formula; indeed, consider quantum stochastic integra

$$
\begin{aligned}
& X(t)=X_{0}+\int_{0}^{t}\left(L_{1}\left(s^{\prime}, \quad+L_{2}(s) d \Lambda(s)+L_{3}(s) d A(s)+L_{4}(s) d s\right)\right. \\
& Y(t)=Y_{0}+\int_{0}^{t}\left(M_{1}(s) d A^{+}(s)+M_{2}(s) d \Lambda(s)+M_{3}(s) d A(s)+M_{4}(s) d s\right)
\end{aligned}
$$

where $L_{j}, M_{j}$ are $\left(h_{S}, \mathcal{M}_{S}\right)$-adapted families of bounded processes for $j=$ $1, \ldots, 4$ and take $k=\mathscr{C}$ so that $\left.h \otimes \hat{k}=\mathscr{C} e_{-\infty}\right) \mathbb{C} e_{0}\left(\mathbb{C} e_{+\infty}\right.$. The Hilbert space $h \otimes \hat{k}$ is unitarily isomorphic to $h \Leftrightarrow h \Leftrightarrow h$. The operators $L, M$ corresponding to the above stochastic integrals and the operator $F$ can be represented by the $3 \times 3$ matrices

$$
L=\left(\begin{array}{ccc}
0 & L_{3} & L_{4} \\
0 & L_{2} & L_{1} \\
0 & 0 & 0
\end{array}\right), M=\left(\begin{array}{ccc}
0 & M_{3} & M_{4} \\
0 & M_{2} & M_{1} \\
0 & 0 & 0
\end{array}\right), F=\left(\begin{array}{ccc}
0 & 0 & \mathbb{1} \\
0 & \mathbb{1} & 0 \\
\mathbb{1} & 0 & 0
\end{array}\right) .
$$

We can then write

$$
X(t)=X_{0}+\Lambda_{L}(t), \quad Y(t)=Y_{0}+\Lambda_{M}(t), \quad Y^{*}(t)=Y_{0}^{*}+\Lambda_{M^{b}}(t) .
$$


In the new notation the Ito formula can be written in the form

$$
d Y^{*}(t) d X(t)=d \Lambda_{M^{b}}(t) d \Lambda_{L}(t)=d \Lambda_{M^{\prime \prime} L}(t) .
$$

This reduces the computation of a product of two stochastic differentials to the computation of a product of two matrices.

The following are fundamental formulae of Boson Fock space quantum stochastic calculus; the proof can be found in [68], and in [74].

Proposition 5.3 Let $L, M \in \mathcal{I}\left(D_{S}, \mathcal{M}_{S}\right)$ and let $X_{0}, Y_{0}$ be operators on $h$ with domain containing $D$. Consider the families $(X(t))_{t \geq 0},(Y(t))_{t \geq 0}$ of operators

$$
X(t)=X_{0}+\Lambda_{L}(t), \quad Y(t)=Y_{0}+\Lambda_{M}(t) .
$$

For each $v, u \in D, f, g \in \mathcal{M}_{S}$ the following formulae hold:

$$
\begin{gathered}
\langle v e(g), X(t) u e(f)\rangle=\left\langle v e(g), X_{0} u e(f)\right\rangle \\
+\int_{0}^{t}\left\langle v\left(e_{-\infty}+g(s)\right) e(g), L(s) u\left(f(s)+e_{+\infty}\right) e(f)\right\rangle d s \\
\langle Y(t) v e(g), X(t) u e(f)\rangle=\left\langle Y_{0} v e(g), X_{0} u e(f)\right\rangle \\
+\int_{0}^{t}\left\{\begin{array}{l}
\left\langle(Y(s)+F M(s) F) v\left(e_{-\infty}+g(s)\right) e(g),\right. \\
\left.(X(s)+L(s)) u\left(f(s)+e_{+\infty}\right) e(f)\right\rangle
\end{array}\right. \\
\left.-\left\langle Y(s) v\left(e_{-\infty}+g(s)\right) e(g), X(s) u\left(f(s)+e_{+\infty}\right) e(f)\right\rangle\right\} d s
\end{gathered}
$$

The above formulae can be written also in coordinate notation. The first, for example, becomes

$$
\begin{aligned}
\langle v e(g), & X(t) u e(f)\rangle=\left\langle v e(g), X_{0} u e(f)\right\rangle \\
& +\sum_{\ell, m \in S} \int_{0}^{t}\left\langle v e(g), L_{m}^{\ell}(s) u e(f)\right\rangle \bar{g}_{\ell}(s) f_{m}(s) d s \\
& +\sum_{\ell \in S} \int_{0}^{t}\left\langle v e(g), L_{+\infty}^{\ell}(s) u e(f)\right\rangle \bar{g}_{\ell}(s) d s \\
& +\sum_{m \in S} \int_{0}^{t}\left\langle v e(g), L_{m}^{-\infty}(s) u e(f)\right\rangle f_{m}(s) d s \\
& +\int_{0}^{t}\left\langle v e(g), L_{+\infty}^{-\infty}(s) u e(f)\right\rangle d s
\end{aligned}
$$

Coherent vectors in the above formulae are used as test vectors. Using the $n$-chaos vectors instead the above formula can be written as follows

$$
\left\langle v g^{\otimes n^{\prime}}, X(t) u f^{\otimes n}\right\rangle=\left\langle v e(g), X_{0} u e(f)\right\rangle
$$




$$
\begin{aligned}
& +\sum_{\ell, m \in S} \int_{0}^{t}\left\langle v g^{\otimes\left(n^{\prime}-1\right)}, L_{m}^{\ell}(s) u f^{\otimes(n-1)}\right\rangle \bar{g}_{\ell}(s) f_{m}(s) d s \\
& +\sum_{\ell \in S} \int_{0}^{t}\left\langle v g^{\otimes\left(n^{\prime}-1\right)}, L_{+\infty}^{\ell}(s) u f^{\otimes n}\right\rangle \bar{g}_{\ell}(s) d s \\
& +\sum_{m \in S} \int_{0}^{t}\left\langle v g^{\otimes n^{\prime}}, L_{m}^{-\infty}(s) u f^{\otimes(n-1)}\right\rangle f_{m}(s) d s \\
& +\int_{0}^{t}\left\langle v g^{\otimes n^{\prime}}, L_{+\infty}^{-\infty}(s) u f^{\otimes n}\right\rangle d s
\end{aligned}
$$

for every pair $n, n^{\prime}$ of non-negative integers with the conventions $g^{\otimes n^{\prime}}=f^{\otimes n}=0$ if $n<0$ and $g^{\otimes 0}=f^{\otimes 0}=e(0)$.

The fundamental formulae of quantum stochastic calculus can be used to prove the following useful estimate of quantum stochastic integrals.

Corollary 5.4 Under the assumptions of Proposition 5.3 we have

$$
\begin{array}{r}
\|(X(t)-X(s)) u e(f)\|^{2} \leq 2 \mathrm{e}^{\|f\|^{2}} \exp \left(\int_{s}^{t}\left(1+\|f(r)\|^{2}\right) d r\right) \\
\cdot \int_{s}^{t}\left\|L(r) u\left(e_{+\infty}+f(r)\right) e(f)\right\|^{2} d r
\end{array}
$$

for each $t, s \in] 0,+\infty[$ with $0 \leq s \leq t$.

We finish this preliminary section by recalling the basic results on quantum stochastic differential equations. Since the solution cannot commute with the coefficient $L \in \mathbb{B}$ we shall distinguish between left (resp. right) quantum stochastic differential equations

$$
d X(t)=X(t) d \Lambda_{L}(t), \quad\left(\text { resp. } d Y(t)=d \Lambda_{L}(t) Y(t)\right)
$$

where the solution acts by left (resp. right) multiplication on the stochastic differential. The above equations have to be interpreted as

$$
X(t)=X_{0}+\int_{0}^{t} X(s) d \Lambda_{L}(s), \quad\left(\text { resp. } Y(t)=Y_{0}+\int_{0}^{t} d \Lambda_{L}(s) Y(s)\right)
$$

where $X_{0}, Y_{0}$ are bounded operators on $h$ (acting on $\mathcal{H}$ by standard ampliation) and $(Y(t))_{t \geq 0},(X(t))_{t \geq 0}$ are $\left(h, \mathcal{M}_{S}\right)$-regular adapted families of operators.

We refer to [68], and [74] for the proof of the following result

Proposition 5.5 For each $M, L \in \mathbb{B}$ and $U_{0}, V_{0} \in \mathcal{B}(h)$ there exists $\left(h, \mathcal{M}_{S}\right)$ regular adapted families of operators $(U(t))_{t \geq 0},(V(t))_{t \geq 0}$ satisfying the quantum stochastic differential equations

$$
\begin{aligned}
d U(t) & =d \Lambda_{M}(t) U(t), & & U(0)=U_{0}, \\
d V(t) & =V(t) d \Lambda_{L}(t), & V(0) & =V_{0} .
\end{aligned}
$$

Moreover: 
1. $(U(t))_{t \geq 0}$ is the unique $\left(h, \mathcal{M}_{S}\right)$-regular adapted family of operators satisfying (5.10),

2. $(V(t))_{t \geq 0}$ is the unique $\left(h, \mathcal{M}_{S}\right)$-regular adapted family of operators such that

$$
\sup _{0 \leq s \leq t .\|u\| \leq 1} \sup _{0 V(s) u e(f) \|<\infty}
$$

for each $t>0$ and each $f \in k$ satisfying (5.11).

Existence can be shown by the Picard's iteration method. Uniqueness for $(U(t))_{t \geq 0}$ follows immediately from Corollary 5.4 and Gronwall's lemma. Uniqueness for $(V(t))_{t \geq 0}$ can be proved as in [74] (Prop. 26.1). The additional property needed will be called initial space boundedness.

The following proposition (see, for example, [40] Prop. 3.1 p.150) gives a necessary and sufficient condition for $(U(t))_{t \geq 0}$ to be a contractive right cocycle.

Proposition 5.6 Let $M$ be an element of $\mathbb{B}$ and let $(U(t))_{t \geq 0}$ be the unique $\left(h, \mathcal{M}_{S}\right)$-regular adapted family of operators satisfying the right quantum stochastic differential equation

$$
d U(t)=d \Lambda_{M}(t) U(t), \quad U(0)=\mathbb{1} .
$$

The operators $U(t)(t \geq 0)$ are contractions if and only if

$$
F\left(M+M^{b}+M^{b} M\right) \leq 0 .
$$

In this case $(U(t))_{t \geq 0}$ is a right cocycle.

Proof. Assume that (5.13) holds. Consider vectors $\xi$ in $\mathcal{H}$ of the form $\sum_{j=1}^{m} u_{j} e$ $\cdot\left(f_{j}\right)$ with $\left(u_{j}\right)_{j=1}^{m}$ in $h$ and functions $\left(f_{j}\right)_{j=1}^{m}$ in $\mathcal{M}_{S}$ continuous at the point 0 . Applying the Itô formula (5.7) we have

$$
\begin{gathered}
\|U(t) \xi\|^{2}=\|\xi\|^{2}+\int_{0}^{t}\left\langle U(r) \sum_{j} u_{j}\left(e_{-\infty}+f_{j}(r)\right) e\left(f_{j}\right),\right. \\
\left.F\left(M+M^{b}+M^{b} M\right) U(r) \sum_{j} u_{j}\left(e_{-\infty}+f_{j}(r)\right) e\left(f_{j}\right)\right\rangle d r
\end{gathered}
$$

Then the inequality (5.13) implies that the operators $U(t)$ are contractions for all $t \geq 0$.

Conversely, if the operators $U(t)$ are contractions, for all $\left(f_{j}\right)_{j=1}^{m}$ such that 0 is a continuity point for every function $f_{j}$, we have

$$
\begin{gathered}
\left\langle\sum_{j} u_{j}\left(e_{-\infty}+f_{j}(0)\right) e\left(f_{j}\right), F\left(M+M^{b}+M^{b} M\right) \sum_{j} u_{j}\left(e_{-\infty}+f_{j}(0)\right) e\left(f_{j}\right)\right\rangle \\
=\lim _{t \rightarrow 0^{+}} \frac{1}{t}\left(\|U(t) \xi\|^{2}-\|\xi\|^{2}\right) \leq 0
\end{gathered}
$$


This proves that the inequality (5.13) holds.

In this case the right cocycle identity follows by a uniqueness argument as in the paper [58]. Indeed, if the operators $U(t)(t \geq 0)$ are contractions then for every fixed $s \geq 0$ the $\left(h, \mathcal{M}_{S}\right)$-regular adapted family of operators

$$
X(t)=U(t+s), \quad Y(t)=\theta_{s}(U(t)) U(s)
$$

both satisfy the quantum stochastic differential equation (5.10) with the same initial condition, and therefore they coincide.

Taking the adjoint of the operators $U(t)$ we immediately prove the following fact.

Corollary 5.7 Let $L$ be an element of $\mathbb{B}$ and let $(V(t))_{t \geq 0}$ be the unique $\left(h, \mathcal{M}_{S}\right)$ rgular adapted initial space bounded family of operators satisfying the left quantum stochastic differential equation

$$
d V(t)=V(t) d \Lambda_{L}(t), \quad V(0)=\mathbb{1} .
$$

Then the operators $V(t)(t \geq 0)$ are contractions if and only if

$$
F\left(L+L^{b}+L L^{b}\right) \leq 0 .
$$

In this case $(V(t))_{t \geq 0}$ is a left cocycle.

Proof. If the operators $V(t)$ are contractions then the adjoint operators $U(t)=$ $V(t)^{*}$ are also contractions and satisfy the right quantum stochastic differential equation $d U(t)=d \Lambda_{L^{b}}(t) U(t)$. Therefore, by Proposition 5.6, the inequality (5.15) holds.

Conversely, if (5.15) holds, then the unique $\left(h, \mathcal{M}_{S}\right)$-regular adapted initial space bounded family of operators satisfying the right quantum stochastic differential equation $d U(t)=d \Lambda_{L^{b}}(t) U(t)$ with $U(0)=\mathbb{1}$ is contractive. The adjoint family $\left(U(t)^{*}\right)_{t \geq 0}$ is also $\left(h, \mathcal{M}_{S}\right)$-regular adapted, contractive and satisfies (5.15). Since the $\left(h, \mathcal{M}_{S}\right)$-regular adapted family of operators solving this equation is unique, it follows that the operators $V(t)$ are also contractions.

Clearly $(V(t))_{t \geq 0}$ is a left cocycle because it is the adjoint of a right cocycle.

\subsection{Time reversal and dual cocycles}

In this section we define a time reversal on Fock space that transforms a right (resp. left) cocycle into right (resp. left) cocycle. Moreover we give the relation between the quantum stochastic differential equations satisfied by a cocycle and its time reversed counterpart.

Time reversal plays a fundamental role in the study of quantum stochastic differential equations with an unbounded operator $L$ when it is too hard to make sense of a right differential equation and one needs to write a differential 
equation for the adjoint of a left cocycle. In fact, if a left cocycle $V$ satisfies (5.15), then the time reversed of the adjoint right cocycle is again a left cocycle which can be shown to satisfy the left equation (5.15) - roughly speaking - with $L$ replaced by $L^{b}$. by

Let $\rho_{t}$ be the unitary time reversal on the interval $[0, t]$ defined on $L^{2}\left(\mathbb{R}_{+}\right) \aleph_{k}$

$$
\left(\rho_{t} f\right)(s)=\left\{\begin{array}{cc}
f(t-s) & \text { if } s \leq t \\
f(s) & \text { if } s>t
\end{array} .\right.
$$

Let $\Gamma\left(\rho_{t}\right)$ be the operator on $\Gamma\left(L^{2}\left(\mathbb{R}_{+}\right) \otimes k\right)$ defined by second quantization of $\rho_{t}$

$$
\Gamma\left(\rho_{t}\right) e(f)=e\left(\rho_{t} f\right) \text {. }
$$

The operators are self-adjoint and satisfy

$$
\rho_{t} \rho_{t}=\mathbb{1}, \quad \Gamma\left(\rho_{t}\right) \Gamma\left(\rho_{t}\right)=\mathbb{1} .
$$

Let $\mathcal{R}_{t}$ be the operator on $\mathcal{B}$ defined by

$$
\mathcal{R}_{t}: \mathcal{B} \rightarrow \mathcal{B}, \quad \mathcal{R}_{t}(x)=\Gamma\left(\rho_{t}\right) x \Gamma\left(\rho_{t}\right)^{*} .
$$

The following lemma gives a useful relationship between time reversal and shift. Recall that a bounded operator $x$ is called $t$-adapted if it is factorised as in condition 2 of Definition 5.2.

Lemma 5.8 For every $t, s \geq 0$ and every t-adapted bounded operator $V(t)$ in $\mathcal{H}$ we have

$$
\mathcal{R}_{t+s}\left(\mathcal{R}_{t}(V(t))=\theta_{s}(V(t)) .\right.
$$

Proof. For every $v, u \in h$ and $g, f \in L^{2}\left(\mathbb{R}_{+}\right) \otimes k$ we have

$$
\left\langle v e(g), \mathcal{R}_{t+s}\left(\mathcal{R}_{t}(V(t)) u e(f)\right\rangle=\left\langle v e\left(\rho_{t} \rho_{t+s} g\right), V(t) u e\left(\rho_{t} \rho_{t+s} f\right)\right\rangle .\right.
$$

A simple computation yields

$$
\left(\rho_{t}\left(\rho_{t+s} f\right)\right)(r)= \begin{cases}f(s+r) & \text { if } r<t \\ f(t+s-r) & \text { if } t<r<t+s \\ f(r) & \text { if } r>t+s\end{cases}
$$

Therefore we have the identity

$$
\rho_{t}\left(\rho_{t+s} f\right)=\left(\sigma_{-s} f\right) 1_{j 0, \ell \mid}+\left(\rho_{t+s} f\right) 1_{j t, t+s[}+f 1_{] t+s, \infty[} .
$$

By the tensor product factorization of Fock space and $t$-adaptedness of $V(t)$ we write the right-hand side of (5.17) as

$$
\begin{array}{r}
\left\langle v e\left(\left(\sigma_{-s} g\right) 1_{] 0, t[}\right), V(t) u e\left(\left(\sigma_{-s} f\right) 1_{|0, t|}\right)\right\rangle \cdot\left\langle e\left(g 1_{\mid t+s, \infty[}\right), e\left(f 1_{\mid t+s, \infty[}\right)\right\rangle \\
\cdot\left\langle e\left(\left(\rho_{t+s} g\right) 1_{|t, t+s|}\right), e\left(\left(\rho_{t+s} f\right) 1_{|t, t+s|}\right)\right\rangle .
\end{array}
$$


Computing the second and third scalar product we write the right-hand side of (5.17) in the form

$$
\begin{aligned}
\left\langle v e\left(g 1_{|s, t+s|}\right), \Gamma\left(\sigma_{s}\right) V(t) \Gamma\left(\sigma_{-s}\right) u e\left(f 1_{1 s, t+s \mid}\right)\right\rangle \\
\cdot \exp \left(\int_{0}^{s}(\bar{g} f)(r) d r f+\int_{t+s}^{\infty}(\bar{g} f)(r) d r\right) \\
=\left\langle v e(g), \theta_{s}(V(t)) u e(f)\right\rangle .
\end{aligned}
$$

This proves the lemma.

We recall now the notion of dual cocycle due to Journé (see [60] p. 294 and also [68] p. 174).

Proposition 5.9 Let $(V(t))_{t \geq 0}$ be a left (resp. right) cocycle. The family of opcrators $(\tilde{V}(t))_{t \geq 0}$ defined by

$$
\widetilde{V}(t)=\mathcal{R}_{t}\left(V(t)^{*}\right)
$$

is a left (resp. right) cocycle.

Proof. By virtue of the cocycle property, for every $s, t \geq 0$ we have

$$
\begin{aligned}
\tilde{V}(t+s) & =\mathcal{R}_{t+s}\left(V(t+s)^{*}\right) \\
& =\mathcal{R}_{t+s}\left(\theta_{s}\left(V(t)^{*}\right) V(s)^{*}\right) \\
& =\mathcal{R}_{t+s}\left(\theta_{s}\left(V(t)^{*}\right)\right) \mathcal{R}_{t+s}\left(V(s)^{*}\right) .
\end{aligned}
$$

Applying $\mathcal{R}_{t+s}$ to both sides of (5.16) we have

$$
\mathcal{R}_{t}\left(V(t)^{*}\right)=\mathcal{R}_{t+s}\left(\theta_{s}\left(V(t)^{*}\right)\right) .
$$

The same identity yields also

$$
\mathcal{R}_{t+s}\left(V(s)^{*}\right)=\mathcal{R}_{t+s}\left(\mathcal{R}_{s}\left(\mathcal{R}_{s}\left(V(s)^{*}\right)\right)\right)=\theta_{t}\left(\mathcal{R}_{s}\left(V(s)^{*}\right)\right) .
$$

This proves the lemma.

Definition 5.10 The cocycle $(\tilde{V}(t))_{t \geq 0}$ defined by (5.18) is called dual cocycle of the cocycle $(V(t))_{t \geq 0}$.

We now study the relationship between a cocycle satisfying a left quantum stochastic differential equation and its dual.

Lemma 5.11 Let $t, s$ be two non-negative real numbers and let $X(s)$ (resp. $Y(t))$ be an $s$-adapted (resp. t-adapted) bounded operator. For every $u, v \in h$ and every $f, g \in \mathcal{M}_{S}$ we have

$$
\left\langle Y(t) v e(g), \theta_{t}\left(\mathcal{R}_{s}(X(s))\right) u e(f)\right\rangle=\left\langle Y(t) v e\left(\rho_{t, t+s} g\right), \theta_{t}(X(s)) u e\left(\rho_{t, t+s} f\right)\right\rangle
$$

where $\rho_{t, t+s}$ is the time reversal operator on the interval $[t, t+s]$ defined by

$$
\left(\rho_{t, t+s} f\right)(r)= \begin{cases}f(2 t+s-r) & \text { if } t<r<t+s \\ f(r) & \text { otherwise. }\end{cases}
$$


Proof. Since the homomorphisms $\theta_{t}$ and $\mathcal{R}_{s}$ are normal it suffices to prove the lemma for the factorised operators

$$
X(s)=X_{0} \otimes X_{s}, \quad Y(t)=Y_{0} \otimes Y_{t}
$$

where $X_{0}, Y_{0}$ are bounded operators in the initial space and $X_{s}$ (resp. $Y_{t}$ ) is a bounded operator in the factor $\Gamma\left(L^{2}(0, s) \otimes k\right)$ (resp. $\left.\Gamma\left(L^{2}(0, t) \otimes k\right)\right)$ of the Fock space $\Gamma\left(L^{2}\left(\mathbb{R}_{+}\right) \otimes k\right)$.

In this case the left-hand side of (5.19) can be written as the product of the factors $\left\langle Y_{0} v, X_{0} u\right\rangle,\left\langle Y_{t} e\left(g 1_{[0, t]}\right), e\left(f 1_{[0, t]}\right)\right\rangle$ and

$$
\begin{array}{r}
\left\langle e\left(g 1_{[t, \infty[}\right), \Gamma\left(\sigma_{t}\right) \Gamma\left(\rho_{s}\right) X_{s} \Gamma\left(\rho_{s}\right) \Gamma\left(\sigma_{-t}\right) e\left(f 1_{[t, \infty]}\right)\right\rangle \\
=\left\langle e\left(\rho_{s} \sigma_{-t}\left(g 1_{[t, \infty]}\right)\right), X_{s} e\left(\rho_{s} \sigma_{-t}\left(f 1_{[t, \infty]}\right)\right)\right\rangle
\end{array}
$$

A simple computation shows that

$$
\rho_{s} \sigma_{-t}\left(g 1_{[t, \infty]}\right)=\sigma_{-t} \rho_{t, t+s} g, \quad \rho_{s} \sigma_{-t}\left(f 1_{[t, \infty]}\right)=\sigma_{-t} \rho_{t, t+s} f .
$$

Therefore we have

$$
\begin{aligned}
\left\langlee \left(\rho_{s} \sigma_{-t}\right.\right. & \left.\left.\left(g 1_{[t, \infty]}\right)\right), X_{s} e\left(\rho_{s} \sigma_{-t}\left(f 1_{[t, \infty]}\right)\right)\right\rangle \\
& \left.=\left\langle e\left(\sigma_{-t} \rho_{t, t+s} g\right)\right), X_{s} e\left(\sigma_{-t} \rho_{t, t+s} f\right)\right\rangle \\
& =\left\langle e\left(\left(\rho_{t, t+s} g\right) 1_{[t, \infty]}\right), \theta_{t}\left(X_{s}\right) e\left(\left(\rho_{t, \ell+s} f\right) 1_{[\ell, \infty]}\right)\right\rangle .
\end{aligned}
$$

The product of this with $\left\langle Y_{t} e\left(g 1_{[0, t]}\right), e\left(f 1_{[0, t]}\right)\right\rangle$ is equal to

$$
\left\langle Y_{t} v e\left(\rho_{t, t+s} g\right), \theta_{t}\left(X_{s}\right) u e\left(\rho_{t, t+s} f\right)\right\rangle .
$$

Multiplication by the initial space factor $\left\langle Y_{0} v, X_{0} u\right\rangle$ then yields (5.19).

Proposition 5.12 Let $L$ be an element of $\mathbb{B}$ satisfying the inequality (5.15) and let $(V(t))_{t \geq 0}$ be the unique $\left(h, \mathcal{M}_{S}\right)$-regular adapted contractive left cocycle $(V(t))_{t \geq 0}$ satisfying (5.14). Then the dual cocycle $(\tilde{V}(t))_{t \geq 0}$ satisfies the left quantum stochastic differential equation

$$
d \widetilde{V}(t)=\widetilde{V}(t) d \Lambda_{L^{b}}(t), \quad \tilde{V}(0)=\mathbb{1} .
$$

Proof. By virtue of Proposition 5.9, for every $t, s \geq 0$ we have

$$
\widetilde{V}(t+s)-\widetilde{V}(t)=\tilde{V}(t) \theta_{t}(\tilde{V}(s)-\mathbb{1}) .
$$

Therefore, applying (5.19), we obtain

$$
\begin{aligned}
& \langle v e(g),(\tilde{V}(t+s)-\tilde{V}(t)) u e(f)\rangle \\
= & \left\langle\widetilde{V}(t)^{*} v e(g), \theta_{t}\left(\mathcal{R}_{s}\left(V(s)^{*}-\mathbb{1}\right)\right) u e(f)\right\rangle \\
= & \left\langle\widetilde{V}(t)^{*} v e\left(\rho_{t, t+s} g\right), \theta_{t}\left(V(s)^{*}-\mathbb{1}\right) u e\left(\rho_{t, t+s} f\right)\right\rangle
\end{aligned}
$$


The action of $\theta_{t}$ on a stochastic integral can be computed explicitly as follows

$$
\begin{aligned}
\theta_{t}\left(V(s)^{*}-\mathbb{1}\right) & =\theta_{t}\left(\int_{0}^{s} d \Lambda_{L^{b}}(r) V(r)^{*}\right) \\
& =\int_{t}^{t+s} d \Lambda_{L^{u}}(r) \theta_{t}\left(V(r-t)^{*}\right) .
\end{aligned}
$$

Therefore, by the first fundamental formula of quantum stochastic calculus (5.6), we have

$$
\begin{aligned}
& \langle v e(g),(\tilde{V}(t+s)-\tilde{V}(t)) u e(f)\rangle \\
= & \left\langle\tilde{V}(t)^{*} v e\left(\rho_{t, t+s} g\right), \int_{t}^{t+s} d \Lambda_{L^{b}}(r) \theta_{t}\left(V(r-t)^{*}\right) u e\left(\rho_{t, t+s} f\right)\right\rangle \\
= & \int_{t}^{t+s}\left\langle\tilde{V}(t)^{*} v\left(e_{-\infty}+g(2 t+s-r)\right) e\left(\rho_{t, t+s} g\right),\right. \\
& \left.L^{b} \theta_{t}\left(V(r-t)^{*}\right) u\left(f(2 t+s-r)+\epsilon_{+\infty}\right) e\left(\rho_{t, t+s} f\right)\right\rangle d r .
\end{aligned}
$$

Dividing by $s$ and letting $s$ tend to 0 , for every $t$ which is a continuity point for both $g$ and $f$ we have

$$
\frac{d}{d t}\langle v e(g), \tilde{V}(t) u e(f)\rangle=\left\langle v\left(e_{-\infty}+g(t)\right) e(g), \tilde{V}(t) L^{b} u\left(f(t)+c_{+\infty}\right) e(f)\right\rangle .
$$

This completes the proof.

\subsection{Quantum stochastic differential equations}

In this section we recall the main results on left quantum stochastic differential equations of the form (5.14) with a possibly unbounded operator $L$ giving in particular necessary and sufficient conditions in order the operaturs $V(t)$ to be isometries, coisometries or unitaries. These extend the results of [37] [50], [52] for special classes of operators $L$.

Several results show that "good" operator cocycles satisfy a quantum stochastic differential equation (sce, for example [7], [40], [57]). A full characterization (a quantum analogue of the classical Stone's theorem on strongly continuous unitary groups), however, is not arailable.

As a first step we complete the study of the case when $L$ is bounded.

Proposition 5.13 Let $L \in \mathbb{B}$ and let $(V(t))_{t \geq 0}$ be the unique $\left(h, M_{S}\right)$-regular adapted initial space bounded process solving the quantum stochastic differentiat equation

$$
d V(t)=V(t) d \Lambda_{L}(t), \quad V(0)=\mathbb{1} .
$$

Then: 
1. the operators $(V(t))_{t \geq 0}$ are contractions if and only if one of the following equivalent inequalities hold

$$
\left(L+L^{b}+L L^{b}\right) F \leq 0, \quad F\left(L+L^{b}+L^{b} L\right) \leq 0,
$$

2. the operators $(V(t))_{t \geq 0}$ are isometries if and only if

$$
L+L^{b}+L^{b} L=0,
$$

3. the operators $(V(t))_{t \geq 0}$ are coisometries if and only if

$$
L+L^{b}+L L^{b}=0,
$$

4. the operators $(V(t))_{t \geq 0}$ are unitary if and only if

$$
L+L^{b}+L^{b} L=0, \quad L+L^{b}+L L^{b}=0 .
$$

Proof. Clearly the operators $V(t)(t \geq 0)$ are contractions if and only if the first inequality (5.21) holds by Corollary 5.7 .

The equivalence of the second inequality (5.21) and contractivity of the operators $V(t)$ can be shown in the same way considering the dual cocycle $\widetilde{V}$. In fact contractivity of $\widetilde{V}$ is equivalent to that of $V$ and the dual cocycle satisfies a left quantum stochastic differential equation with $L^{b}$ instead of $L$ by Proposition 5.12 .

We prove then 2. For every $v, u \in h$ and every $f, g \in \mathcal{M}_{S}$ formula (5.6) yields

$$
\begin{aligned}
& \left\langle\tilde{V}(t)^{*} v e(g), \tilde{V}(t)^{*} u e(f)\right\rangle=\langle v e(g), u e(f)\rangle \\
& +\int_{0}^{t}\left\langle\tilde{V}(s)^{*} v\left(e_{-\infty}+g(s)\right) e(g), F\left(L+L^{b}+L^{b} L\right) \tilde{V}(s)^{*} u\left(e_{-\infty}+f(s)\right) e(f)\right\rangle d s
\end{aligned}
$$

Since $\tilde{V}(t)^{*}=\mathcal{R}_{t}(V(t))$ this shows that the operators $(V(t))_{t \geq 0}$ are isometries if and only if (5.22) holds.

The third statement can be proved in the same way considering the right cocycle $\left(V(t)^{*}\right)_{t \geq 0}$.

The fourth statement follows immediately from 2 and 3.

Remark. When $L$ is the operator (5.5) we have

$$
L+L^{b}+L^{b} L=\left(\begin{array}{ccc}
0 & L_{3}+L_{1}^{*}+L_{1}^{*} L_{2} & L_{4}+L_{4}^{*}+L_{1}^{*} L_{1} \\
0 & L_{2}+L_{2}^{*}+L_{2}^{*} L_{2} & L_{1}+L_{3}^{*}+L_{2}^{*} L_{1} \\
0 & 0 & 0
\end{array}\right)
$$

In this case, condition (5.22), (resp. (5.23)) is equivalent to the well-known condition for the solution of a quantum stochastic differential equation to be isometric (resp. coisometric) found in the seminal paper [58] by R.L. Hudson and K.R. Parthasarathy. 
Note that, if the operators $\left(L_{k}\right)_{k=1}^{4}$ are bounded (for simplicity), the identity $L_{4}+L_{4}^{*}+L_{1}^{*} L_{1}=0$ implies that

$$
L_{4}+\frac{1}{2} L_{1}^{*} L_{1}
$$

has the form $i H$ where $H$ is bounded self-adjoint operator. Therefore, the choice

$$
L_{1}=L_{2}=L_{3}=0, \quad L_{4}=i H
$$

yields a group of unitary operators $(V(t))_{t \geq 0}$ satisfying the Schroedinger equation

$$
d V(t)=i H V(t) d t
$$

1. Tis serise (5.20) is a generalization of the Schroedinger cquation.

The following propositions motivate partially the assumptions under which we shall st dy the quantum stochastic differential equation (5.20) with an unbounded operator $L$.

Let us recall first the following (see, for example, 60] or 68] Ch. VI, Sect. $12)$.

Proposition 5.14 Let $(V(t))_{t \geq 0}$ be a contractive left cocycle in $\mathcal{H}$. Then the family $(P(t))_{t \geq 0}$ of operators on $h$ defined by

$$
P(t)=\mathbb{E}_{0 \mathfrak{j}}[V(t)]
$$

is a contraction semigroup in $h$. If the cocycle is strongly continuous then the semigroup $(P(t))_{t \geq 0}$ is also.

Proof. Since $\left(\theta_{t}\right)_{t \geq 0}$ is a covariant shift and $V$ is a cocycle for crery $t, s \geq 0$ we have

$$
\begin{aligned}
P(t+s) & =\mathbb{E}_{0]}[V(t+s)] \\
& =\mathbb{E}_{0]}\left[V(s) \theta_{s}(V(t))\right] \\
& =\mathbb{E}_{0 !}\left[V(s) \mathbb{E}_{s]}\left[\theta_{s}(V(t))\right]\right] \\
& =\mathbb{E}_{0 !}\left[V(s) \theta_{s}\left(\mathbb{E}_{0]}[V(t)]\right)\right]
\end{aligned}
$$

Notice that $\theta_{s}(P(t))=P(t)$ because the shift $\left(\theta_{l}\right)_{t \geq 0}$ leaves invariant operators on the initial space. Hence we have

$$
P(t+s)=\mathbb{E}_{0,}[V(s)] P(t)=P(s) P(t) .
$$

Therefore $(P(t))_{t \geq 0}$ is a semigroup. Moreover, for every $u \in h$, we have

$$
\begin{aligned}
\|(P(t+s)-P(t)) u\| & =\sup _{v \in h,\|v\|=1}|\langle v,(P(t+s)-P(t)) u\rangle| \\
& =\sup _{v \in h,\|v\|=1}|\langle v e(0),(V(t+s)-V(t)) u e(0)\rangle| \\
& \leq\|(V(t+s)-V(t)) u e(0)\| .
\end{aligned}
$$


Strong continuity of $V$ implies then the strong continuity of $(P(t))_{t \geq 0}$. A similar argument shows that the semigroup is also contractive.

¿From now on we shall always suppose that $(P(t))_{t \geq 0}$ is strongly continuous. The infinitesimal generator $G$ of $P$ is defined as the set of $u \in h$ such that the limit

$$
\lim _{t \rightarrow 0^{+}} t^{-1}(P(t) u-u)
$$

exists in the strong (or equivalently weak) topology of $h$.

The following natural hypothesis on the operator $L$ that will be in force throughout the rest of this chapter.

\section{Hypothesis L}

1. There exists an operator $G$ which is the infinitesimal generator of a strongly continuous contraction semigroup in $h$ and a core $D$ for $G$ such that the domain of the operator $L$ contains the domain $D_{S, G}$

$$
D_{S, G}=\left\{w e_{-\infty}+u f+v e_{+\infty} \mid w \in h, u, v \in D, f \in k\right\} .
$$

2. for all $u, v \in D, w \in h$, we have

$$
\begin{gathered}
L w e_{-\infty}=0, \quad E_{+\infty} L\left(v e_{+\infty}+u f\right)=0 \\
\langle w, G v\rangle=\left\langle w e_{-\infty}, L v e_{+\infty}\right\rangle
\end{gathered}
$$

3. for all $x \in D_{S, G}$ we have

$$
\langle F x, L x\rangle+\langle L x, F x\rangle+\langle F L x, L x\rangle \leq 0 .
$$

Remark. The above hypothesis implies that the operators $G$ and $L_{+\infty}^{\ell}$ satisfy the hypothesis $\mathbf{A}$ introduced in Chapter 3. Indeed, for every $u \in D$, taking $x=u e_{+\infty},(5.27)$ reads as

$$
\langle G u, u\rangle+\sum_{\ell=1}^{\infty}\left\langle L_{+\infty}^{\ell} u, L_{+\infty}^{\ell} u\right\rangle+\langle u, G u\rangle \leq 0 .
$$

Since $D$ is a core for $G$, the operators $L_{+\infty}^{\ell}$ can be extended to $D(G)$ so that the above in equality holds also for $u \in D(G)$.

Remark. Since the coefficient $L$ is unbounded but the solutions we shall construct are contractive the left quantum stochastic differential equation will be interpreted through quantum stochastic integrals defined on vectors of the form $u e(f)$ with $u \in D$. We shall stress this fact by speaking of quantum stochastic differential equations in $\mathcal{D}_{S}$.

Under the hypothesis $\mathbf{L}$ an operator $L$ in $h \otimes \hat{k}$ can be approximated by a sequence of bounded operators $\left(L_{n}\right)_{n \geq 1}$ which are the "infinitesimal generators" of contractive cocycles. 
For every integer $n \geq 1$, let $R(n ; G)$ be the bounded operator $(n \mathbb{1}-G)^{-1}$. Note that the adjoint operator $G^{*}$ is the infinitesimal generator of the dual contraction semigroup and we have $R\left(n ; G^{*}\right)=R(n ; G)^{*}$. Moreover the wollknown properties of resolvent operators relations for all $w \in h, v \in D(G)$ yield

$$
\lim _{n \rightarrow \infty} n R(n ; G) w=w, \quad \lim _{n \rightarrow \infty} n G R(n ; G) v=G v .
$$

Proposition 5.15 Let $L$ be an operator in $h \otimes \hat{k}$ satisfying the hypothesis $\mathrm{L}$. For each $n \geq 1$ let $I_{n}, L_{n}$ be the operators in $h \otimes \hat{k}$ with domain $\mathcal{D}_{S}$ defined by

$$
I_{n}=n R(n ; G) E_{-\infty}+E+n R(n ; G) E_{+\infty}, \quad L_{n}=I_{n}^{*} L I_{n} .
$$

Then the operator $L_{n}$ has a bounded extension which is an element of $\mathbb{B}$ satisfling the inequalities (5.21) and its uniform norm can estimated by

$$
\left\|L_{n}\right\| \leq 2(n+3 \sqrt{n}+1) .
$$

Moreover, for all $\xi \in D_{S}$, we have

$$
\lim _{n \rightarrow \infty} L_{n} \xi=L \xi .
$$

Proof. The operator $L_{n}$ satisfies the inequality (5.27). Indeed it suffices to write (5.27) for vectors of the form $I_{n} x$ with $x \in D_{S, G}$ (the vector $I_{n} x$ belongs to the domain of $L$ by the well-known properties of the operators $R(n ; G)$ and the remark after the hypothesis $\mathbf{L}$ ) and use the commutation of the opcrator $F$ with the operators $I_{n}$ and $I_{n}^{*}$.

Moreover, because of (5.26), in order to prove that $L_{n}$ is bounded, it suffices to estimate the norm of $L_{n} \xi$ for vectors $\xi$ in $h \times \hat{k}$ of the form $\sum_{j=1}^{J} u_{j}\left(f_{j}+e_{+\infty}\right)$ with $u_{j} \in D, f_{j} \in \mathcal{M}_{S}$ for all $j$. In this case we have

$$
\left\|L_{n} \xi\right\| \leq\left\|E L_{n} E \xi\right\|+\left\|E_{-\infty} L_{n} E \xi\right\|+\left\|E L_{n} E_{+\infty} \xi\right\|+\left\|E_{-\infty} L_{n} E_{+\infty} \xi\right\|
$$

The norm of $E_{-\infty} L_{n} E_{+\infty}$ can be estimated using the third identity (5.26). We have in fact, for each $v, u \in D$,

$$
\left\langle v e_{-\infty} \cdot E_{-\infty} L_{n} E_{+\infty} u e_{+\infty}\right\rangle=n^{2}\langle R(n ; G) v, G R(n ; G) u\rangle
$$

Then, using the identity $G R(n ; G)=n R(n ; G)-\mathbb{1}$ and the contractivity of $n R(n ; G)$, we can estimate the norm of $G R(n ; G)$ by 2. Therefore we obtain the inequality

$$
\left|\left\langle v e_{-\infty}, E_{-\infty} L_{n} E_{+\infty} u e_{+\infty}\right\rangle\right| \leq 2 n\|v\| \cdot\|u\|
$$

which implies

$$
\left\|E_{-\infty} L_{n} E_{+\infty}\right\| \leq 2 n .
$$

Taking a vector $x \in D_{S}$ in the range of $E$ from the inequality (5.27) for $L$. . we obtain immediately the estimates

$$
\left\|E+E L_{n} E\right\| \leq 1, \quad\left\|E L_{n} E\right\| \leq 2 .
$$


Consider now a vector $x=u e_{+\infty}$ with $u \in D$. The inequality (5.27) for $L_{n}$ yields

$$
2 n^{2} \Re e\langle R(n ; G) u, G R(n ; G) u\rangle+\left\|E L_{n} E_{+\infty} u e_{+\infty}\right\|^{2} \leq 0
$$

Hence, using again the equality $G R(n ; G)=n R(n ; G)-\mathbb{1}$, and the contractivity of $n R(n ; G)$ we get the inequality

$$
\left\|E L_{n} E_{+\infty} u e_{+\infty}\right\|^{2} \leq 4 n\left\|u e_{+\infty}\right\|^{2}
$$

which implies

$$
\left\|E L_{n} E_{+\infty}\right\| \leq 2 \sqrt{n}
$$

To estimate the norm of $E_{-\infty} L_{n} E$ we apply inequality (5.27) for $L_{n}$ with vectors $x$ of the form $x_{0}+\lambda v e_{+\infty}$ with $x_{0}$ finite linear combination of vectors in $D_{S}$ in the range of $E, v \in D$ and $\lambda \in \mathbb{R}$. It turns out that the number

$$
\begin{aligned}
& \left\langle x_{0}, L x_{0}\right\rangle+\left\langle L x_{0}, x_{0}\right\rangle+\left\langle L x_{0}, E L x_{0}\right\rangle \\
+ & \lambda^{2}\left(\left\langle v e_{-\infty}, L_{n} v e_{+\infty}\right\rangle+\left\langle L_{n} v e_{+\infty}, v e_{-\infty}\right\rangle+\left\langle L_{n} v e_{+\infty}, F L_{n} v e_{+\infty}\right\rangle\right) \\
+ & 2 \lambda \Re e\left\langle L x_{0}, F L_{n} v e_{+\infty}\right\rangle+2 \lambda \Re e\left(\left\langle L x_{0}, v e_{-\infty}\right\rangle+\left\langle L_{n} v e_{+\infty}, x_{0}\right\rangle\right) \\
= & 2 \Re e\left\langle x_{0}, L x_{0}\right\rangle+\left\|L x_{0}\right\|^{2}+\lambda^{2}\left(2 \Re e\left\langle v e_{-\infty}, L_{n} v e_{+\infty}\right\rangle+\left\|E L_{n} v e_{+\infty}\right\|^{2}\right) \\
+ & 2 \lambda \Re e\left(\left\langle L x_{0}, F L_{n} v e_{+\infty}\right\rangle+\left\langle L x_{0}, v e_{-\infty}\right\rangle+\left\langle L_{n} v e_{+\infty}, x_{0}\right\rangle\right)
\end{aligned}
$$

is real negative for every $\lambda \in \mathbb{R}$. Let

$$
\begin{aligned}
a & =-2 \Re e\left\langle v e_{-\infty}, L_{n} v e_{+\infty}\right\rangle-\left\|E L_{n} E_{+\infty} v e_{+\infty}\right\|^{2} \\
c & =-2 \Re e\left\langle x_{0}, L x_{0}\right\rangle-\left\|E L x_{0}\right\|^{2}=-\left\|(E+E L) x_{0}\right\|^{2}+\left\|x_{0}\right\|^{2} \\
b & =\operatorname{Re}\left\{\left\langle v e_{-\infty}, E_{-\infty} L_{n} E x_{0}\right\rangle+\left\langle\left(E+E L_{n} E\right) x_{0}, E L_{n} E_{+\infty} v e_{+\infty}\right\rangle\right\}
\end{aligned}
$$

Therefore we have the inequality

$$
a \lambda^{2}-2 b \lambda+c \geq 0
$$

Applying (5.27) for $L_{n}$ with $x=v e_{+\infty}$ and $x=x_{0}$ separately we find that $a$ and $c$ are nonnegative. Moreover the estimates (5.31) and (5.32) yield

$$
a \leq 4 n\|v\|^{2}, \quad c \leq\left\|x_{0}\right\|^{2} .
$$

Therefore $|b| \leq \sqrt{a c}$ and we obtain the estimate

$$
\left|\Re e\left\langle v e_{-\infty}, E_{-\infty} L_{n} E x_{0}\right\rangle\right| \leq 2 \sqrt{a c}+\left|\left\langle\left(E+E L_{n} E\right) x_{0}, E L_{n} E_{+\infty} v e_{+\infty}\right\rangle\right|
$$

Multiplying $v$ by a complex number of modulus 1 such that the scalar product in the right-hand side becomes real, from (5.32), (5.33), (5.34) we obtain the inequality

$$
\left|\left\langle v e_{-\infty}, E_{-\infty} L_{n} E x_{0}\right\rangle\right| \leq 4 \sqrt{n}\|v\| \cdot\left\|x_{0}\right\|
$$

for all $v \in D$ which implies

$$
\left\|E_{-\infty} L_{n} E\right\| \leq 4 \sqrt{n}
$$


Using (5.32), (5.33), (5.34) and the above inequality from (5.30) we obtain the claimed estimate for the norm of $L_{n}$. Clearly $L_{n} \in \mathbb{B}$ because of (5.26).

Now (5.29) can be proved by decomposing $L_{n} \xi$ as we did to obtain (5.30) and using the properties of resolvent operators $\left(R\left(n ; G^{*}\right)\right)_{n \geq 1}$. In fact, for every $u \in D$ and $f \in h$, we have

$$
\begin{aligned}
\left\|\left(E_{-\infty} L_{n} E_{+\infty}-E_{-\infty} L E_{+\infty}\right) u e_{+\infty}\right\|^{2} & =\left\|\left(n^{2} R\left(n ; G^{*}\right) G R(n ; G)-G\right) u\right\|^{2} \\
\left\|\left(E L_{n} E_{+\infty}-E L E_{+\infty}\right) u e_{+\infty}\right\|^{2} & =\left\|E L(n R(n ; G) u-u) e_{+\infty}\right\|^{2} \\
\leq-2 \Re e\langle n R(n ; G) u-u, G(n R(n ; G) u-u)\rangle & \\
\left(E_{-\infty} L_{n} E-E_{-\infty} L E\right) u f & =E_{-\infty}\left(n R\left(n ; G^{*}\right) L-L\right) u f \\
\left(E L_{n} E-E L E\right) u f & =0 .
\end{aligned}
$$

This completes the proof.

Proposition 5.16 Let $L$ be an operator on h $\otimes \hat{k}$ satisfying hypothesis $\mathbf{L}$ and let $\left(L_{n}\right)_{n \geq 1}$ be a sequence of elements of $\mathbb{I B}$ satisfying (5.27) such that, for all $\xi \in D_{S}$, we have

$$
\lim _{n \rightarrow \infty} L_{n} \xi=L \xi
$$

For all integer $n$ let $V_{n}$ be the unique $\left(h, M_{S}\right)$-adapted regular contractive solution of the guantum stochastic differential cunation in $h$.

$$
d V_{n}(t)=V_{n}(t) d \Lambda_{L_{n}}(t) . \quad V_{n}(0)=\mathbb{1} .
$$

There exist a subsequence $\left(n_{m}\right)_{m \geq 1}$ and operators $V(t)$ defincd by

$$
V(t)=w-\lim _{m \rightarrow \infty} V_{n, .}(t)
$$

satisfying the quantum stochastic differentiul equation in $D_{S}$

$$
d V(t)=V(t) d . I_{L}(t) . \quad V(0)=\mathbb{1} .
$$

Proof. For each $n \geq 1$ and each $u \in D . f \in \mathcal{M}_{S}$ by the Ito formula (5.7). Corollary 5.4 and contractivity of $V_{n}(t)$ we have

$$
\left\|\left(V_{n}(t)-V_{n}(s)\right) u c(f)\right\|^{2} \leq 2 \exp \left(1+2\|f\|^{2}\right) \int_{s}^{t} \mid L_{n} u\left(f(r)+e_{+\infty}\right) \|^{2} d r .
$$

Let $N(f)$ be the number of nonzero $f_{j}$ with $j \in S$ and let $c^{\prime}(u, f)$ be the constant

$$
\max _{n \in N}\left\{\max _{j \in\left\{k \in S \mid f_{k} \neq 0\right\}}\left\{\left\|L_{n} u e_{j}\right\|^{2}\right\},\left\|L_{n} u e_{+\infty}\right\|^{2},\|u\|^{2}, 1\right\} .
$$

A straightforward computation yields

$$
\left\|L_{n} u\left(f(r)+e_{+x}\right)\right\|^{2} \leq(1+. V(f)) c^{\prime}(u, f)\left(1+\|f(r)\|^{2}\right) .
$$


Therefore, for each $y \in \mathcal{H}, u \in D, f \in \mathcal{M}_{S}$, the sequence of functions on $[0,+\infty]$

$$
t \mapsto\left\langle y, V_{n}(t) u e(f)\right\rangle
$$

is equicontinuous and equibounded. Hence, by the Ascoli-Arzelà theorem, there exists a subsequence uniformly convergent on every bounded interval of $[0,+\infty[$. The diagonalisation argument and the scparability of $\mathcal{H}$ allow to find a subsequence $\left(V_{n_{m}}\right)_{m \geq 1}$ weakly convergent to an $\left(h, \mathcal{M}_{S}\right)$-adapted contractive process $V$.

Lower semicontinuity of the norm with respect to the weak convergence and (5.37) imply that $V$ is strongly continuous. Moreover, for all $N$, by (5.6) we have

$$
\begin{aligned}
& \left\langle v e(g), V_{n_{m}}(t) u e(f)\right\rangle=\langle v e(g) \cdot u \in(f)\rangle \\
& \quad+\int_{0}^{t}\left\langle v\left(e_{-\infty}+g(r)\right) \cdot(g) \cdot V_{n_{m}}(r) L_{n_{m}} u\left(f(r)+r_{+\infty}\right) c(f)\right\rangle(r)
\end{aligned}
$$

Letting $m$ tend to infinity, contractions $V_{n_{m}}(s)$ converge weakly to $V(s)$ an! vectors $L_{n n_{m}} u\left(f(r)+e_{+\infty}\right)$ converge strongly to $L u\left(f(r)+e_{+\infty}\right)$. Therefore $L_{k}$ desired conclusion follows.

The above propositions allow us to prove immediately the foll wing

Theorem 5.17 Let $L$ be an operator on $h \hat{k}$ sutisfying hypothesi.s $\mathbf{L}$. Then there exists a $\left(D, \mathcal{M}_{S}\right)$-adapted regular contractive solution of the quantum stochastic differential equation (5.96).

Arguing by induction on the number $n$ of the $n$-th chaos of the Fock space as in [39] can prove the following uniqueness result due to A. Mobari (669').

Theorem 5.18 Let $L$ be an operator on $h \quad \hat{k}$ satisfying hypothen $L$. Then the (h. $\left.\mathcal{M}_{S}\right)$-adapted regular contractice solution of the quantum stochuster differential equation (5.36) is unique.

Proof. Let $(X(t))_{t \geq 0}$ be the difference of two (h.MS)-adapres mgular contractive solution of (5.36). Applying the first fundamental formata (5.9) we have

$$
\begin{aligned}
& \left\langle v g^{\otimes n^{\prime}}, X(t) u f^{\otimes n}\right\rangle=\int_{0}^{t}\left\langle v g^{\otimes n^{\prime}} \cdot Y(s) G u f^{\otimes n}\right\rangle d s \\
& +\sum_{\ell, m \in S} \int_{0}^{t}\left\langle u g^{(2(n-1)} \cdot X(*) L_{,}^{t} u f(n-1)\right\rangle \bar{g}(s) f_{m}(s) d s \\
& +\sum_{\ell \in S} \int_{0}^{\ell}\left\langle\lg \left(n^{\prime}-1\right) \cdot X(s) L_{+\infty}^{\ell} u j^{\phi n}\right\rangle \bar{g}_{\ell}(s) d s \\
& +\sum_{m \in S} \int_{0}^{t}\left\langle v g^{\omega n^{\prime}}, X(s) L_{m}^{-\infty} u f^{\omega(n-1)}\right\rangle f_{m}(s) d s
\end{aligned}
$$


for every $u, v \in D$ and every pair $n, n^{\prime}$ of integer numbers with the convention $g^{\otimes n^{\prime}}=f^{\otimes n}=0$ if $n<0$ and $g^{\otimes 0}=f^{\otimes 0}=e(0)$.

We prove that the left-hand side vanishes by induction on $n+n^{\prime}$.

For every $\lambda>0$ the bilinear form on $h$

$$
(v, u) \rightarrow \int_{0}^{\infty} \exp (-\lambda t)\langle v e(0), X(t) u e(0)\rangle d t
$$

is bounded because $\|X(s)\| \leq 2$ and

$$
\left|\int_{0}^{\infty} \exp (-\lambda t)\langle v e(0), X(t) u e(0)\rangle d t\right| \leq 2 \lambda^{-1}\|v\| \cdot\|u\| .
$$

Hence there exists a bounded operator $R_{\lambda}$ in $h$ such that

$$
\left\langle v, R_{\lambda} u\right\rangle=\int_{0}^{\infty} \exp (-\lambda t)\langle v e(0), X(t) u e(0)\rangle d t .
$$

The first fundamental formula (5.9) for $n=n^{\prime}=0, u, v \in D$, yields

$$
\begin{aligned}
\lambda\left\langle v, R_{\lambda} u\right\rangle & =\lambda \int_{0}^{\infty} \exp (-\lambda t) d t \int_{0}^{t}\langle v e(0), X(s) G u e(0)\rangle d s \\
& =\lambda \int_{0}^{\infty}\langle v e(0), X(s) G u e(0)\rangle d s \int_{s}^{\infty} \exp (-\lambda t) d t \\
& =\left\langle v, R_{\lambda} G u\right\rangle
\end{aligned}
$$

We have then

$$
R_{\lambda}(\lambda \mathbb{1}-G) u=0
$$

for every $u \in D$. Since $D$ is a core for $G$, the linear manifold $(\lambda \mathbb{1}-G)(D)$ is dense in $h$. Thus $R_{\lambda}$ vanishes. Therefore $\langle v e(0), X(t) u e(0)\rangle$ also vanishes for every $t \geq 0$.

This establishes our claim for $n+n^{\prime}=0$. Suppose it has been established for every $n, n^{\prime}$ such that $n+n^{\prime} \leq m$. Then, for every $n, n^{\prime}$ with $n+n^{\prime}=m+1$, the induction hypothesis allows us to write formula (5.9) as

$$
\left\langle v g^{\otimes n^{\prime}}, X(t) u f^{\otimes n}\right\rangle=\int_{0}^{t}\left\langle v g^{\otimes n^{\prime}}, X(s) G u f^{\otimes n}\right\rangle d s .
$$

The same argument we used in the case when $n=n^{\prime}=0$ shows then that $\left\langle v g^{\otimes n^{\prime}}, X(t) u f^{\otimes n}\right\rangle$ vanishes for every $t \geq 0$.

This completes the induction argument and the proof.

Uniqueness allows us to deduce the cocycle property as in the paper [58].

Corollary 5.19 Let $L$ be an operator on $h \otimes \hat{k}$ satisfying hypothesis $\mathrm{L}$. Then the unique $\left(h, \mathcal{M}_{S}\right)$-adapted regular contractive solution of the quantum stochastic differential equation (5.36) is a left cocycle. 
Proof. In fact, since the operators $V(t)(t \geq 0)$ are contractions then for every fixed $s \geq 0$ the $\left(h, \mathcal{M}_{S}\right)$-regular adapted family of operators

$$
X(t)=V(t+s), \quad Y(t)=V(s) \theta_{s}(V(t))
$$

satisfy both the quantum stochastic differential equation (5.36) with the same initial condition. Therefore they coincide.

\subsection{Unitary solutions}

In this section we will study conditions on the operator $L$ (satisfying the hypothesis $\mathbf{L}$ ) for the solution $(V(t))_{t \geq 0}$ of $(5.36)$ to be a family of isometries (resp. coisometries, unitaries).

Proposition 5.13 suggests necessary condition: the left-hand side of (5.27) vanishes for $x \in D_{S}$. This condition, as $\mathbf{A A}$ for quant um dynamical semigroups, unfortunately is not sufficient. However we will show that, under the hypothesis $\mathbf{L}$, the operators $(V(t))_{t \geq 0}$ are isometries if and only if an associated quantum dynamical semigroup is identity preserving.

This reduces our problem to the problem studied in Chapter 3, Sections 5 and 6 .

With a given contractive cocycle $V$, by Theorem 2.24, we can associate quantum dynamical semigroup $\mathcal{T}=\left(\mathcal{T}_{t}\right)_{t \geq 0}$ on $\mathcal{B}(h)$ defined by

$$
\begin{aligned}
\left\langle v, \mathcal{T}_{t}(x) u\right\rangle & =\langle V(t) v e(0), x V(t) u e(0)\rangle \\
& =\left\langle\widetilde{V}(t)^{*} v e(0), x \widetilde{V}^{*}(t) u e(0)\right\rangle
\end{aligned}
$$

for all $t \geq 0, x \in \mathcal{B}(h), v, u \in D$. Here the second identity follows from the properties of time reversal operators $\mathcal{R}_{t}$ and the fact that $\mathcal{R}_{t} e(0)=e(0)$.

In this section the following hypothesis on the operator $L$ will be in force

Hypothesis LL have

The operator $L$ in $h \otimes \hat{k}$ satisfies the hypothesis $\mathbf{L}$ and, for all $x \in D_{S, G}$ we

$$
\langle F x, L x\rangle+\langle L x, F x\rangle+\langle F L x, L x\rangle=0 .
$$

Lemma 5.20 Let $L$ be an operator in $h \otimes \hat{k}$ satisfying the hypothesis LL. For every $\eta \in[0,1[$ consider the operators in $h \otimes \hat{k}$

$$
\mathbb{1}^{(\eta)}=\eta E_{-\infty}+E+\eta E_{+\infty}, \quad L^{(\eta)}=\mathbb{1}^{(\eta)} L \mathbb{1}^{(\eta)}+\left(1-\eta^{2}\right) E_{-\infty} L E_{+\infty}
$$

The operator $L^{(\eta)}$ satisfies the hypothesis $\mathbf{L}$.

Proof. The operators $\mathbb{1}^{(\eta)}$ and $F$ commute and $D_{S, G}$ is invariant under $\mathbb{1}^{(\eta)}$. Therefore $L^{(\eta)}$ obviously satisfies 1 and 2 of hypothesis $\mathbf{L}$. 
Moreover, for every $x \in D_{S, G}$, we have

$$
\begin{aligned}
& \left\langle F x, L^{(\eta)} x\right\rangle+\left\langle L^{(\eta)} x, F x\right\rangle+\left\langle F L^{(\eta)} x, L^{(\eta)} x\right\rangle \\
= & \left\langle F \mathbb{1}^{(\eta)} x, L \mathbb{1}^{(\eta)} x\right\rangle+\left\langle L \mathbb{1}^{(\eta)} x, F \mathbb{1}^{(\eta)} x\right\rangle+\left\langle F L \mathbb{1}^{(\eta)} x, L \mathbb{1}^{(\eta)} x\right\rangle \\
+ & \left(1-\eta^{2}\right)\left(\left\langle F x, E_{-\infty} L E_{+\infty} x\right\rangle+\left\langle E_{-\infty} L E_{+\infty} x, F x\right\rangle\right) \\
= & \left(1-\eta^{2}\right)\left(\left\langle F E_{+\infty} x, L E_{+\infty} x\right\rangle+\left\langle L E_{+\infty} x, F E_{+\infty} x\right\rangle\right) \\
= & -\left(1-\eta^{2}\right)\left\langle L E_{+\infty} x, E L E_{+\infty} x\right\rangle \leq 0 .
\end{aligned}
$$

This completes the proof.

The above lemma allows us to state the following

$\hat{I}$.oposition 5.21 Let $L$ be an operator in $h \otimes \hat{k}$ satisfying the hypothesis $\mathbf{L L}$. F.r every $\eta \in\left[0,1\left[\right.\right.$ let $V^{(\eta)}$ be the unique regular $\left(h, \mathcal{M}_{S}\right)$-adapted contractive princess satisfying the quantum stochastic differential equation

$$
d V^{(\eta)}(t)=V^{(\eta)}(t) d \Lambda_{L^{(\eta)}}(t), \quad V^{(\eta)}(0)=\mathbb{1}
$$

in $h$ and let $\mathcal{T}^{(1)}$ be the associated quantum dynamical semigroup. The following fincts hold:

1. for every $t \geq 0$

$$
w-\lim _{\eta \rightarrow 1} V^{\prime \prime}(t)=V(t)
$$

where $(V(t))_{t \geq 0}$ is the unique solution of (5.36),

2. for every $t \geq 0$ and every positive operator $x \in \mathcal{B}(h)$ the operators $\left(\mathcal{T}_{t}^{(\eta)}(x)\right.$

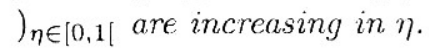

Proof. As in the proof of Proposition 5.16, applying Corollary 5.4 we can find the estimate

$$
\left\|\left(V^{(\eta)}(t)-V^{(\eta)}(s)\right) u e(f)\right\|^{2} \leq c(u, f) \int_{s}^{t}\left(1+\|f(r)\|^{2}\right) d r
$$

for each $u \in D$ and $f \in \mathcal{M}$ where $c(u, f)$ is a constant independent of $\eta$. The equicontinuity and diagonalization argument in the proof of Proposition 5.16 can be used also here to show that for every sequence $\left(\eta_{n}\right)_{n \geq 0}$ converging to 1 there exists a subsequence $\left(\eta_{n_{m}}\right)_{m \geq 0}$ such that the limit

$$
w-\lim _{m \rightarrow \infty} V^{\left(\eta_{n_{m}}\right)}(t)
$$

exists for every $t \geq 0$.

Moreover, it can be shown as in the proof of the same proposition, that the limit satisfies (5.36). Therefore it coincides with the unique solution of (5.36) by Theorem 5.18 . 
Summing up: every sequence $\left(V^{\left(\eta_{n}\right)}\right)_{n \geq 0}$ admits a subsequence which converges weakly to the unique solution of (5.36). This proves the claim 1.

Let $\mathcal{T}^{(\eta)}$ be the quantum dynamical semigroup associated with $V^{(\eta)}$ via (5.38) and let $(P(t))_{\iota \geq 0}$ be the strongly continuous contraction semigroup on $h$ generated by $G$. A straightforward computation yields

$$
\begin{aligned}
& \frac{d}{d s}\left\langle V^{(\eta)}(s) P(t-s) u e(0), x V^{(\eta)}(s) P(t-s) u e(0)\right\rangle \\
= & \eta^{2} \sum_{\ell \in S}\left\langle V^{(\eta)}(s) L_{+\infty}^{\ell} P(t-s) u e(0), x V^{(\eta)}(s) L_{+\infty}^{\ell} P(t-s) u e(0)\right\rangle
\end{aligned}
$$

for every $u \in D$ and $x \in \mathcal{B}(h)$. Therefore, integrating on $[0, t]$, we have (dropping the index $+\infty$ of $\left.L_{+\infty}^{\ell}\right)$

$$
\begin{aligned}
\left\langle u, \mathcal{T}_{t}^{(\eta)}(x) u\right\rangle & =\langle P(t) u, x P(t) u\rangle \\
& +\eta^{2} \sum_{\ell \in S} \int_{0}^{t}\left\langle L^{\ell} P(t-s) u, \mathcal{T}_{s}^{(\eta)}(x) L^{\ell} P(t-s) u\right\rangle d s
\end{aligned}
$$

For every $\eta_{2} \leq \eta_{1}<1$ and every positive operator $x \in \mathcal{B}(h)$ we have then

$$
\begin{aligned}
& \left\langle u,\left(\mathcal{T}_{t}^{\left(\eta_{1}\right)}(x)-\mathcal{T}_{t}^{\left(\eta_{2}\right)}(x)\right) u\right\rangle \\
= & \left(\eta_{1}^{2}-\eta_{2}^{2}\right) \sum_{\ell \in S} \int_{0}^{t}\left\langle L^{\ell} P(t-s) u, \mathcal{T}_{s}^{\left(\eta_{1}\right)}(x) L^{\ell} P(t-s) u\right\rangle d s \\
+ & \eta_{2}^{2} \sum_{\ell \in S} \int_{0}^{t}\left\langle L^{\ell} P(t-s) u,\left(\mathcal{T}_{s}^{\left(\eta_{1}\right)}(x)-\mathcal{T}_{s}^{\left(\eta_{2}\right)}(x)\right) L^{\ell} P(t-s) u\right\rangle d s \\
\geq & \eta_{2}^{2} \sum_{\ell \in S} \int_{0}^{t}\left\langle L^{\ell} P(t-s) u,\left(\mathcal{T}_{s}^{\left(\eta_{1}\right)}(x)-\mathcal{T}_{s}^{\left(\eta_{2}\right)}(x)\right) L^{\ell} P(t-s) u\right\rangle d s .
\end{aligned}
$$

Estimating the norm of $\mathcal{T}_{t}^{\left(\eta_{1}\right)}(x)-\mathcal{T}_{t}^{\left(\eta_{2}\right)}(x)$ by $2\|x\|$ and computing the integral

$$
\begin{aligned}
\left\langle u,\left(\mathcal{T}_{t}^{\left(\eta_{1}\right)}(x)-\mathcal{T}_{t}^{\left(\eta_{2}\right)}(x)\right) u\right\rangle & \geq-2\|x\| \eta_{2}^{2} \sum_{\ell \in S} \int_{0}^{t}\left\|L^{\ell} P(t-s) u\right\|^{2} d s \\
& =-2\|x\| \eta_{2}^{2}\left(\|u\|^{2}-\|P(t) u\|^{2}\right) \\
& \geq-2\|. x\| \eta_{2}^{2}\|u\|^{2}
\end{aligned}
$$

Iterating the estimate and computing the integral we obtain

$$
\begin{aligned}
& \left\langle u,\left(\mathcal{T}_{t}^{\left(\eta_{1}\right)}(x)-\mathcal{T}_{t}^{\left(\eta_{2}\right)}(x)\right) u\right\rangle \\
\geq & \eta_{2}^{2} \sum_{\ell \in S} \int_{0}^{t}\left\langle L^{\ell} P(t-s) u,\left(\mathcal{T}_{s}^{\left(\eta_{1}\right)}(x)-\mathcal{T}_{s}^{\left(\eta_{2}\right)}(x)\right) L^{\ell} P(t-s) u\right\rangle d s \\
\geq & -2\|x\| \eta_{2}^{4} \sum_{\ell \in S} \int_{0}^{t}\left\|L^{\ell} P(t-s) u\right\|^{2} d s \\
\geq & -2 \eta_{2}^{4}\|x\| \cdot\|u\|^{2} .
\end{aligned}
$$


Therefore, iterating $n$ times, we have

$$
\left\langle u,\left(\mathcal{T}_{t}^{\left(\eta_{1}\right)}(x)-\mathcal{T}_{t}^{\left(\eta_{2}\right)}(x)\right) u\right\rangle \geq-2 \eta_{2}^{2 n}\|x\| \cdot\|u\|^{2} .
$$

The statement 2 follows then letting $n$ tend to infinity.

Theorem 5.22 Let $L$ be an operator in $h \& \hat{k}$ satisfying the hypothesis $\mathbf{L L}$ and let $(V(t))_{I \geq 0}$ be the unique solution of (5.36). The quantum dynamical semigroup associated with the cocycle $V$ coincides with the minimal quantum dynamical semigroup $\mathcal{T}^{(\mathrm{min})}$ associated with the operators $G, L_{+\infty}^{\ell}$.

Proof. Wr use the notation of the proof of Proposition 5.21. Let $x$ be a positive operator $\mathcal{B}(h), u$ a vector in $h$ and $t \geq 0$. Letting $\eta$ tend to $l$ it turns out that the limit quamum. dynamical semigroup satisfies the equations (3.16) and (3.15). $\therefore$ vover. for $: \in\{0,1\}, t \geq 0$, by the same argument of the proof of Proposition 3.21 , we have

$$
\mathcal{T}_{t}^{(\eta)}(x) \leq \mathcal{T}_{t}^{(\min )}(x) .
$$

Therefore the limit quantum dynamical semigroup coincides with the minimal quantum dynamical semigroup $\mathcal{T}^{\text {(nin) }}$ associated with the operators $G, L_{+\infty}^{l}$ by the minimality of $\mathcal{T}^{(\mathrm{min})}$ (see Theorem 3.22).

Let $\mathcal{T}$ be the quantum dynamical semigroup associated with the cocycle $V$. The second fundamental formula of quantum stochastic calculus (5.7) shows immediately that $\mathcal{T}$ satisfies the equations (3.16) and (3.15). Therefore, by virtue of Theorem 3.22 , we have

$$
\mathcal{T}_{t}(x) \geq \mathcal{T}_{t}^{\left(\min ^{\prime}\right.}(x) .
$$

On the other haid the lower semicontinuity of the norm with respect to weak convergence yiekis

$$
\begin{aligned}
\left\langle u, \mathcal{T}_{t}(x) u\right\rangle & =\left\|x^{1 / 2} V(t) u e(0)\right\|^{2} \\
& \leq \liminf _{\eta \rightarrow 1^{-}}\left\|x^{1 / 2} V^{(\eta)}(t) u e(0)\right\|^{2} \\
& =\liminf _{\eta-1^{-}}\left\langle u, \mathcal{T}_{t}^{(\eta)}(x) u\right\rangle \\
& =\left\langle u \cdot \mathcal{T}_{t}^{(\min )}(x) u\right\rangle .
\end{aligned}
$$

This completes the proof.

We can now give necessary and sufficient conditions for the operators $V(t)$ to be isometries.

Theorem 5.23 Let $L$ be an operator in $h \otimes \hat{k}$ satisfying the hypothesis LL and let $(V(t))_{t \geq 0}$ be the unique contractive cocycle satisfying (5.36). The following conditions are equivalent: 
1. t've operators $V(t)$ are isometries for every $t \geq 0$,

2. for each $v, u \in D$ and each $t \geq 0$ we have

$$
\langle V(t) v e(0), V(t) u e(0)\rangle=\langle v, u\rangle,
$$

3. the quantum dynamical semigroup associated with the cocycle $V$ is conservative.

Proof. Clearly the first condition implies the second one.

By virtue of Theorem 5.22, the quantum dynamical semigroup associated with the cocycle $V$ coincides with the minimal quantum dynamical semigroup $\mathcal{T}^{(\min )}$ associated with the operators $G$ and $L_{+\infty}^{\ell}$. Therefore we have

$$
\langle V(t) v e(0), x V(t) u e(0)\rangle=\left\langle v, \mathcal{T}_{t}^{(\min )}(x) u\right\rangle
$$

for every $v, u \in D, x \in \mathcal{B}(h)$ and $t \geq 0$. Taking $x=\mathbb{1}$ it is clear that 2 and 3 are equivalent.

To complete the proof it suffice then to show that 2 implies 1 . We will prove by induction that for each $m \geq 0, n, n^{\prime} \in \mathbb{N}$ with $n+n^{\prime}=m, v, u \in D$, $g, f \in \mathcal{M}_{S}$ we have

$$
\left\langle V(t) v g^{\otimes n^{\prime}}, V(t) u f^{\otimes n}\right\rangle=\delta_{n^{\prime}, n}\left\langle(\cdot u\rangle\langle g \cdot f\rangle^{n} .\right.
$$

This claim is clearly true for $m=0$ because of condition 2. Suppose it has been established for all $n, n^{\prime}$ with $n+n^{\prime}=m$ and take $n \cdot n^{\prime}$ such that $n+n^{\prime}=m+1$.

Writing the Ito formula (5.7) of Boson Fock space quantum stochastic calculus using $n$-chaos vectors and taking into the account the induction hypothesis. we obtain the equation

$$
\begin{aligned}
\left\langle V(t) v g^{\otimes n^{\prime}}, V(t) u f^{\otimes n}\right\rangle=\delta_{n^{\prime}, n}\langle u, u\rangle\langle g, f\rangle^{n} \\
+\int_{0}^{t}\left\{\left\langle V(s) v g^{\otimes n^{\prime}}, V(s) G u f^{\otimes n}\right\rangle+\left\langle V(s) G v g^{\otimes n^{\prime}}, V(s) u f^{\otimes n}\right\rangle\right. \\
\left.+\left\langle V(s) E L v e_{+\infty} g^{\otimes n^{\prime}}, V(s) E L u e_{+\infty} f^{\otimes n}\right\rangle\right\} d s
\end{aligned}
$$

Let $\lambda>0$ fixed and (as in the proof of Theorem 5.18) define a bounded operator $R_{\lambda}$ in $h$ by

$$
\left\langle v, R_{\lambda} u\right\rangle=\int_{0}^{\infty} \exp (-\lambda t)\left\langle V(t) v g^{* n^{\prime}}, V(t) u f \propto n\right\rangle d t
$$

A simple computation (again as in the proof of Theorem 5.18) yields

$$
\lambda\left\langle v, R_{\lambda} u\right\rangle=\delta_{n^{\prime}, n}\langle v, u\rangle\langle g, f\rangle^{n}+\left\langle v, £\left(R_{\lambda}\right) u\right\rangle
$$

i.e., since $£(\mathbb{1})=0$ (in the form sense)

$$
\mathcal{E}\left(R_{\lambda}-c \mathbb{1}\right)=\lambda\left(R_{\lambda}-c \mathbb{1}\right)
$$


where $c=\lambda^{-i} \delta_{n^{\prime}, n}\langle v, u\rangle\langle g, f\rangle^{n}$.

Condition 2 (or the equivalent condition 3) and Proposition 3.31 imply then

$$
R_{\lambda}=\lambda^{-1} \delta_{n^{\prime}, n}\langle g, f\rangle^{n} \mathbb{1}
$$

Hence (5.42) follows for $n+n^{\prime}=m+1$. This completes the proof.

Theorem 5.23 together with the results of Chapter 3 allow us to construct a large class of isometric cocycles. Proposition 5.12 suggests a simple way to determine whether a cocycle is coisometric. In fact this question could be answered applying Theorem 5.23 to the dual cocycle.

The following proposition gives a natural condition on the operator $L$ allowing to find the quantum stochastic differential equation satisfied by the dual cocych.

Hypothesis LLD We say that the operator $L$ satisfies the hypothesis LLD if it satisfin the hypothesis $\mathbf{L L}$ and:

1. there exists a core $\widetilde{D}$ for $G^{*}$ such that $\widetilde{D}_{S, G}$. is contained in the domain of $F L^{*} F$,

2. the restriction $\widetilde{L}$ of $F L^{*} F$ to $\widetilde{D}_{S, G^{*}}$ satisfies the hypothesis $\mathbf{L}$ with $\widetilde{D}$ and $G^{*}$ replacing $D$ and $G$,

3. ther exists a sequence $\left(L_{n}\right)_{n \geq 1}$ of elements of $\mathbb{B}$ such that

$$
\begin{array}{ll}
s-\lim _{n \rightarrow \infty} L_{n} \xi=L \xi ; & \text { for every } \xi \in D_{S, G^{*}} \\
s-\lim _{n \rightarrow \infty} L_{n}^{b} \tilde{\xi}=\tilde{L} \tilde{\xi}, & \text { for every } \tilde{\xi} \in D_{S, G^{*}}
\end{array}
$$

Proposition 5.24 Suppose that the operator $L$ in $h \otimes \hat{k}$ satisfies the hypothesis LLD. Let $V$ and $Z$ be the unique cocycles satisfying the quantum stochastic differential equations

$$
\begin{array}{llrl}
d V(t)=V(t) d \Lambda_{L}(t), & V(0)=\mathbb{1}, & \text { in } \mathcal{D}_{S} \\
d Z(t)=Z(t) d \Lambda_{\widetilde{L}}(t), & & Z(0)=\mathbb{1} & \text { in } \widetilde{\mathcal{D}}_{S}
\end{array}
$$

Then $Z$ coincides with the dual cocycle $\tilde{V}$ of $V$.

Proof. Let $\left(L_{n}\right)_{n \geq 1}$ be the sequence of bounded approximations of $L$. For cvery $n \geq 1$ let $V_{n}$ be the unique cocycle satisfying the quantum stochastic differential equation

$$
d V_{n}(t)=V_{n}(t) d \Lambda_{L_{n}}(t), \quad V_{n}(0)=\mathbb{1}
$$

By virtue of Proposition 5.12 the dual cocycle $\widetilde{V}_{n}$ satisfies the quantum stochastic differential equation

$$
d \widetilde{V}_{n}(t)=\widetilde{V}_{n}(t) d \Lambda_{L_{n}^{b}}(t), \quad \widetilde{V}_{n}(0)=\mathbb{1} .
$$


By the hypothesis LLD we can apply Proposition 5.16 and find subsequences (that we denote as the given sequence for simplicity) such that

$$
w-\lim _{n \rightarrow \infty} V_{n}(t)=V(t), \quad w-\lim _{n \rightarrow \infty} \widetilde{V}_{n}(t)=\widetilde{V}(t)
$$

Then it can be shown as in the proof of Proposition 5.12 that the cocycle $\widetilde{V}$ satisfies the same quantum stochastic differential equation satisfied by the cocycle $Z$.

Therefore, by Theorem 5.18, the two cocycles coincide.

The following Lemma shows that the sequence $\left(L_{n}\right)_{n \geq 1}$ considered in Proposition 5.15 can often be used to check the hypothesis LLD.

Lemma 5.25 Let $L$ be an operator in $h \otimes \hat{k}$ satisfying the hypothesis $\mathbf{L}$. Suppose that there exists a domain $\widetilde{D}$ which is a core for $G^{*}$ such that:

1. the domain of the operator $L^{*}$ contains $\widetilde{D}_{S}$,

2. we have $\widetilde{L} \subseteq\left(F L^{*} F\right)$,

3. for all $u \in D$ and all $n \geq 1, R(n ; G) u$ belongs to the domain of $G^{*}$ and the sequence $\left(G^{*} R(n ; G) u\right)_{n \geq 1}$ converges.

Then the operator $L$ satisfies the hypothesis LLD.

Proof. Let $\left(L_{n}\right)_{n \geq 1}$ be the sequence (5.28).

We show that, under 1,2 and 3 the sequence $\left(L_{n}^{b}\right)_{n>0}$ converges strongly to $\widetilde{L}$ on $\widetilde{D}_{S}$. In fact, for all $\xi \in \widetilde{D}_{S}$ we have

$$
\begin{aligned}
L_{n}^{b} \xi= & E L_{n}^{b} E \xi+E_{-\infty} L_{n}^{b} E \xi+E L_{n}^{b} E_{+\infty} \xi+E_{-\infty} L_{n}^{b} E_{+\infty} \xi \\
= & (E L E)^{*} \xi+n R\left(n ; G^{*}\right) F E_{+\infty} L^{*} E \xi+\left(E_{-\infty} L_{n} E\right)^{*} F \xi \\
& +E_{-\infty} L_{n}^{b} E_{+\infty}
\end{aligned}
$$

Clearly the first two terms converge strongly when $n$ tends to infinity. The vector $E_{+\infty} \xi$ can be written in the form $u e_{+\infty}$ with $u \in \widetilde{D}$; for all $n, m \in \mathbb{N}$, hence we have

$$
\left\|\left(E_{-\infty} L_{n}^{b} E_{+\infty}-E_{-\infty} L_{m}^{b} E_{+\infty}\right) \xi\right\|=\left\|\left(G_{n}\right)^{*} u-\left(G_{m}\right)^{*} u\right\| .
$$

Since $\left(G_{n}\right)^{*} u=n^{2} R\left(n ; G^{*}\right) G^{*} R(n ; G) u$ and $\widetilde{D}$ is contained in $D\left(G^{*}\right)$, the limit as $n$ tends to infinity of the fourth term exists. The third term can be written in the form

$$
E L_{n}^{b} E_{+\infty}=\left(E_{-\infty} L_{n} E\right)^{*} F=-(E+E L E)^{*} E L_{n} E_{+\infty} F
$$

where the second identity follows from the equality (5.39). This shows that the sequence $\left(L_{n}^{b}\right)_{n>0}$ is strongly convergent. To show that the limit coincides with 
$\widetilde{L}$ on $\widetilde{D}_{S}$ consider $y \in \widetilde{D}_{S}, x \in D(\widetilde{L})$. Because of 2 we have then,

$$
\begin{aligned}
\lim _{n \rightarrow \infty}\left\langle y, L_{n}^{b} x\right\rangle & =\lim _{n \rightarrow \infty}\left\langle L_{n} F y, F x\right\rangle \\
& =\langle L F y, F x\rangle \\
& =\left\langle y, F L^{*} F x\right\rangle \\
& =\langle y, \widetilde{L} x\rangle
\end{aligned}
$$

This completes the proof.

\subsection{Inner quantum flows}

In this section, following [41], we show how to construct a class of quantum . [arkov processes with unbounded infinitesimal generator using unitary solulions of quantum stochastic differential equations. Moreover we give a general condition in order the restrictions to an abelian sub-*-algebra to be commutative.

Definition 5.26 . Let $\mathcal{A}$ be a unital sub-*algebra of $\mathcal{B}(h)$. A quantum flow on $\mathcal{A}$ is a family $j=\left(j_{t}\right)_{t} \geq 0$ of identity preserving ${ }^{*}$-homomorphisms from $\mathcal{A}$ into $\mathcal{B}(\mathcal{H})$ such that:

1. for all $x \in \mathcal{A}, j_{0}(x)=x$,

2. for all $x \in \mathcal{A},\left(j_{t}(x)\right)_{t \geq 0}$ is an $\left(h, \mathcal{M}_{S}\right)$-regular adapted process,

3. there exists a sub- ${ }^{*}$ algebra $\mathcal{A}_{\infty}$ of $\mathcal{A}$ dense in $\mathcal{A}$ for the norm $\|\cdot\|_{\infty}$ and structure maps $\theta_{m}^{\ell}: \mathcal{A}_{\infty} \mapsto \mathcal{A}$ such that the quantum stochastic differential equation

$$
d j_{t}(x)=\sum_{\ell, m \in \bar{S}} j_{t}\left(\theta_{m}^{\ell}(x)\right) d \Lambda_{\ell}^{m}(t), \quad j_{0}(x)=x
$$

in $\mathcal{D}_{S}$ is satisfied for all $x \in \mathcal{A}_{\infty}$.

We refer to [68], [74] for results on quantum flows with bounded structure maps in particular, the existence theorem of M. Evans [34].

Definition 5.27 Let $\mathcal{A}$ be a commutative sub-*algebra of $\mathcal{B}(h)$. A quantum flow $\left(j_{t}\right)_{t \geq 0}$ on $\mathcal{A}$ is commutative if, for all $x, y \in \mathcal{A}$ and all $s, t \geq 0$, we have

$$
\left[j_{s}(y), j_{s+t}(x)\right]=0 .
$$

In the following we shall assume

\section{Hypothesis LLF}

An operator $L$ in $h \otimes \hat{k}$ satisfies the hypothesis LLF if:

1. it satisfies LLD, 
2. $\widetilde{D}$ is a dense submanifold of $D$,

3. $V, \widetilde{V}$ are the unique unitary solutions of the quantum stochastic differential equations

$$
\begin{aligned}
& d V(t)=V(t) d \Lambda_{L}(t), \quad V(0)=\mathbb{1}, \quad \text { in } \mathcal{D}_{S}, \\
& d \widetilde{V}(t)=\tilde{V}(t) d \Lambda_{\tilde{L}}(t), \quad \tilde{V}(0)=\mathbb{1}, \quad \text { in } \tilde{\mathcal{D}}_{S} .
\end{aligned}
$$

Note that $V, \widetilde{V}$ are strong limits of cocycles $V_{n}, \widetilde{V}_{n}$ satisfying the quantum stochastic differential equation in $\mathcal{H}$

$$
\begin{array}{llrl}
d V_{n}(t) & =V_{n}(t) d \Lambda_{L_{n}}(t), & & V_{n}(0)=\mathbb{1} \\
d \widetilde{V}_{n}(t)=\widetilde{V}_{n}(t) d \Lambda_{\widetilde{L}_{n}}(t), & & \widetilde{V}_{n}(0)=\mathbb{1}
\end{array}
$$

by Proposition 5.24.

Theorem 5.28 Let $L$ be an operator in $h \otimes \hat{k}$ satisfying the hypothesis LLF and let $\mathcal{A}$ be a sub-*-algebra of $\mathcal{B}(h)$. Moreover let $\mathcal{A}_{\infty}$ be a sub-*-algebra of $\mathcal{A}$ which is dense in $\mathcal{A}$ for the uniform norm $\|\cdot\|_{\infty}$. Suppose that:

1. for all $x \in \mathcal{A}_{\infty}, w \in \widetilde{D}, \ell, m \in \bar{S}$, the strong limit

$$
s-\lim _{n \rightarrow \infty}{ }^{(n)} \theta_{m}^{\ell}(x) w
$$

exists and defines a bounded operator $\theta_{m}^{\ell}(x)$ satisfying, for all $u, v \in \widetilde{D}$,

$$
\begin{aligned}
\left\langle v, \theta_{m}^{\ell}(x) u\right\rangle & =\left\langle F L^{b} F v e_{\ell}, x u e_{m}\right\rangle \\
& +\left\langle v e_{\ell}, x L^{b} u e_{m}\right\rangle+\left\langle F L^{b} F v e_{\ell}, x L^{b} u e_{m}\right\rangle
\end{aligned}
$$

2. for all $x \in \mathcal{A}_{\infty}, \ell, m \in \bar{S}$, we have

$$
\sup _{n}\left\|(n) \theta_{m}^{\ell}(x)\right\|_{\infty}<\infty .
$$

Let $V, \widetilde{V}$ be the unique unitary solutions of the quantum stochastic differential equations (5.44), (5.45) in $\widetilde{\mathcal{D}}_{S}$. For all $t \geq 0$ consider the ${ }^{*}$-homomorphism $j_{t}$ on $\mathcal{A}$ defined by

$$
j_{t}(x)=V(t) x V^{*}(t) .
$$

Then $\left(j_{t}\right)_{t \geq 0}$ is a quantum flow on $\mathcal{A}$ with structure maps given by (5.46) satisfying the quantum stochastic differential equation (5.43).

Proof. Under the present hypotheses we have

$$
s-\lim _{n \rightarrow \infty} V_{n}(t)=V(t), \quad s-\lim _{n \rightarrow \infty} \widetilde{V}_{n}(t)=\widetilde{V}(t), \quad s-\lim _{n \rightarrow \infty} V_{n}^{*}(t)=V^{*}(t)
$$


for all $t \geq 0$. Moreover the cocycles $V$ and $\widetilde{V}$ satisfy quantum stochastic differential equations (5.44), (5.45). For all $n \in \mathbb{N}, u, v \in D, f, g \in \mathcal{M}_{S}$ and all $x \in \mathcal{A}$ we have then

$$
\begin{aligned}
& \left\langle V_{n}^{*}(t) v e(g), x V_{n}^{*}(t) u e(f)\right\rangle=\langle\operatorname{ve}(g), x u e(f)\rangle \\
& \quad+\sum_{\ell, m \in \bar{S}} \int_{0}^{t}\left\langle V_{n}^{*}(s) v e(g),{ }^{(n)} \theta_{m}^{\ell}(x) V_{n}^{*}(s) u e(f)\right\rangle \bar{g}_{\ell}(s) f^{m}(s) d s
\end{aligned}
$$

where, for all $\ell, m \in \bar{S}$, the structure maps ${ }^{(n)} \theta_{m}^{\ell}$ are given by (5.46) with $L$ replaced by $L_{n}$. For all $x \in \mathcal{A}_{\infty}$ the operators ${ }^{(n)} \theta_{m}^{\ell}(x)$ in $h$ are uniformly bounded by condition 2 . Then, because of condition 1 , for each $w \in h$ and each l. $m \in \bar{S}$, we have

$$
\lim _{n \rightarrow \infty}{ }^{(n)} \theta_{m}^{\ell}(x) w=\theta_{m}^{\ell}(x) w .
$$

Hence, by the dominated convergence theorem, taking the limit of both sides of (5.47) we obtain (5.43).

The following results give sufficient conditions in order that the restriction to a commutative sub-*-algebra of $\mathcal{B}(h)$ of a quantum Markov flow in Fock space with unbounded structure maps to be commutative.

Proposition 5.29 Let $\gamma: \mathcal{A}_{\infty} \mapsto \mathcal{A}$ be a linear map such that $(\lambda \mathbb{1}-\gamma)\left(\mathcal{A}_{\infty}\right)$ is dense in $\mathcal{A}$ for the norm $\|\cdot\|_{\infty}$ for all $\lambda$ greater than a fixed positive number $\lambda_{0}$. Let $\left(\mathcal{T}_{t}\right)_{t \geq 0}$ be a family of linear maps $\mathcal{T}_{t}: \mathcal{A} \rightarrow \mathcal{B}(h)$ with the following properties:

1. for all $t \geq 0$ and all $x \in \mathcal{A}$, we have

$$
\left\|\mathcal{T}_{t}(x)\right\|_{\infty} \leq\|x\|_{\infty}
$$

2. for all $x \in \mathcal{A}$ and all $v, u \in \widetilde{D}$ the function $t \mapsto\left\langle v, \mathcal{T}_{t}(x) u\right\rangle$ is continuous,

3. for all $x \in \mathcal{A}_{\infty}, v, u \in \widetilde{D}$ we have

$$
\left\langle v, \mathcal{T}_{t}(x) u\right\rangle=\int_{0}^{t}\left\langle v, \mathcal{T}_{s}(\gamma(x)) u\right\rangle d s .
$$

Then $\mathcal{T}_{t}=0$ for all $t \geq 0$.

Proof. For all $\lambda>0$ let $\widehat{\mathcal{T}}_{\lambda}$ be the operator on $\mathcal{B}(h)$ defined by

$$
\left\langle v, \widehat{T}_{\lambda}(x) u\right\rangle=\int_{0}^{\infty} \exp (-\lambda s)\left\langle v, \mathcal{T}_{s}(x) u\right\rangle d s
$$

for all $x \in \mathcal{A}$ and all $v, u \in \widetilde{D}$. Taking the Laplace transform of both sides of (5.49) and integrating by parts the right-hand side we obtain

$$
\left\langle v, \widehat{\mathcal{T}}_{\lambda}((\lambda \mathbb{1}-\gamma)(x)) u\right\rangle=0,
$$


for all $x \in \mathcal{A}_{\infty}$. This implies that $\widehat{\mathcal{T}}_{\lambda}=0$ because of condition 1 and the density of $(\lambda \mathbb{1}-\gamma)\left(\mathcal{A}_{\infty}\right)$ in $\mathcal{A}$. Hence we obtain $\mathcal{T}_{t}=0$ for all $t \geq 0$ by a well-known property of the Laplace transform.

Remark. If $(\lambda \mathbb{1}-\gamma)\left(\mathcal{A}_{\infty}\right)$ is $\sigma$-weakly dense in $\mathcal{A}$ and the map $x \rightarrow \mathcal{T}_{t}(x)$ satisfying 1,2 and 3 is also $\sigma$-weakly continuous and positive the same conclusion holds.

The following result, obtained in [41], generalises the corresponding result of Parthasarthy and Sinha [75] for quantum flows with bounded structure maps.

Theorem 5.30 Let $\left(j_{t}\right)_{l \geq 0}$ be a quantum flow on $\mathcal{A}$ with structure maps $\left(\theta_{m}^{\ell}\right.$ )$_{\ell, m \in \bar{S}}$ with

$$
\theta_{m}^{\ell}: \mathcal{A}_{\infty} \mapsto \mathcal{A}
$$

satisfying the quantum stochastic differential equation (5.43). Suppose that $\left(\lambda \mathbb{1}-\theta_{+\infty}^{-\infty}\right)\left(\mathcal{A}_{\infty}\right)$ is dense in $\mathcal{A}$ for all $\lambda$ greater than a positive number $\lambda_{0}$. Then the following facts hold:

1. if $\mathcal{A}$ is commutative, then the quantum flow $\left(j_{t}\right)_{t \geq 0}$ is commutative,

2. the adapted family of operators $\left(j_{t}(x)\right)_{t>0}$ is the unique solution of the quantum stochastic differential équation (5.43) for all $x \in \mathcal{A}_{\infty}$.

Proof. Fix $y \in \mathcal{A}$ and $g, f \in \mathcal{M}_{S}$. We will prove that, for all $p, q \in \mathbb{N}$, $s, t \geq 0, v, u \in \widetilde{D}$ and all $x \in \mathcal{A}$ we have

$$
\left\langle v g^{\otimes q},\left[j_{s}(y), j_{s+t}(x)\right] u f^{\otimes p}\right\rangle=0
$$

Let us first consider the case when $n=0$. For all $t \geq 0$ from the homomorphism property of $j_{t}$ we have $\left\|j_{t}(a)\right\|_{\infty} \leq\|a\|_{\infty}$. Hence we can define bounded operators $\mathcal{T}_{t}: \mathcal{A} \rightarrow \mathcal{B}(h)$ by

$$
\left\langle v, \mathcal{T}_{t}(x) u\right\rangle=\left\langle v e(0),\left[j_{s}(y), j_{s+t}(x)\right] u e(0)\right\rangle
$$

Write $j_{s+t}(y)$ as the sum of $j_{s}(x)$ and a stochastic integral on $(0, t)$. Since $j_{s}$ is a *-homomorphism we have

$$
\left[j_{s}(x), j_{s}(y)\right]=j_{s}([x, y])=0 .
$$

Then, applying the Ito formula (5.7), we obtain the equation

$$
\left\langle v, \mathcal{T}_{t}(x) u\right\rangle=\int_{0}^{t}\left\langle v, \mathcal{T}_{r}\left(\theta_{+\infty}^{-\infty}(x)\right) u\right\rangle d r .
$$

and (5.50) follows from Proposition 5.29. The proof can be completed by induction on $n=p+q$ by the same argument of the case $n=0$. 


\subsection{Quantum diffusions}

In this section we construct an inner quantum flow extending the flow of a classical multidimensional diffusions with smooth covariance and drift. This generalises the result obtained in [41] for elliptic diffusions.

Let $(y(t))_{t \geq 0}$ be a $d$-dimensional diffusion, as considered in Chapter 4, Section 2 , satisfying the stochastic differential equation

$$
d y_{j}(t)=\sum_{k=1}^{d} \sigma_{j k}(y(t)) d w_{k}(t)+b_{j}(y(t)) d t, \quad 1 \leq j \leq d
$$

In this section we will always assume that $\sigma$ and $b$ satisfy the hypothesis D in Chapter 4, Section 2. The quantum extension of the semigroup obtained there guides the our construction of the operator cocycle here.

Denote again $a=\sigma^{*} \sigma$.

Let $h=L^{2}\left(\mathbb{R}^{d} ; \widetilde{C}\right)$ and let $D$ and $\widetilde{D}$ be the linear manifold $C_{c}^{\infty}\left(\mathbb{R}^{d} ; \mathscr{C}\right)$ of compact support functions with continuous partial derivatives of all orders. Let $S=\{1, \ldots, d\}$ and $\bar{S}=S \cup\{-\infty,+\infty\}$. For every operator $L$ in $h \otimes \hat{k}$ we denote by $L^{b}$ the restriction to $D_{S}$ of the operator $F L^{*} F$.

Let $\mathcal{A}=C_{l}^{0}\left(\mathbb{R}^{d} ; \mathbb{R}\right)$ and $\mathcal{A}_{\infty}$ be the algebra generated by multiplication operators by a function which is either constant or infinitely differentiable with compact support.

We define now an operator $L$ which is the candidate "infinitesimal generator" of a cocycle $V$ satisfying the quantum stochastic differential equation

$$
d V(t)=V(t) d \Lambda_{L}(t), \quad V(0)=\mathbb{1} .
$$

Denote by $\widetilde{L}$ be the restriction to $D_{S}$ of $F L^{*} F$.

If both the operators $L$ and $\widetilde{L}$ satisfy the hypothesis $\mathbf{L}$ then the following identities hold

$$
\begin{aligned}
& E L E+E L^{*} E+E L E L^{*} E=0 \\
& E L E+E L^{*} E+E L^{*} E L E=0 \\
& E L E_{+\infty}+E L^{b} E_{+\infty}+E L E L^{b} E_{+\infty}=0 \\
& E_{-\infty} L E_{+\infty}+E_{-\infty} L^{b} E_{+\infty}+E_{-\infty} L E L^{b} E_{+\infty}=0
\end{aligned}
$$

Let us consider:

- a $d \times d$ matrix $\gamma$ with entries in $C_{b}^{4}\left(\mathbb{R}^{d} ; \mathbb{R}\right)$ such that $\mathbb{1}+\gamma$ is unitary (real orthogonal),

- a $d$-dimensional vector $\rho$ in $C_{b}^{3}\left(\mathbb{R}^{d} ; \mathbb{R}\right)$,

- a $d$-dimensional vector $\eta$ in $C_{b}^{2}\left(\mathbb{R}^{d} ; \mathbb{R}\right)$ such that

$$
\eta_{j}=b_{j}-\frac{1}{2} \sum_{k=1}^{d}\left(\partial_{k} a_{k j}\right)+\sum_{\ell=1}^{d} \rho_{\ell} \sigma_{\ell j} .
$$


Define the operator $L$, the "infinitesimai generator" of a cocycle $V$, through its "matrix elements" $L_{\ell}^{m}$.

$$
\begin{array}{rlr}
D\left(L_{\ell}^{m}\right)=h & L_{\ell}^{m}=M\left(\gamma_{\ell}^{m}\right) & \text { if } \ell, m \in S \\
D\left(L_{\ell}^{-\infty}\right)=H^{1}\left(\mathbb{R}^{d} ; \mathbb{C}\right) & L_{\ell}^{-\infty}=-\sum_{j=1}^{d} \partial_{j} \sigma_{\ell j}+\rho_{\ell} & \text { if } \ell \in S
\end{array}
$$

where $M\left(\gamma_{\ell}^{m}\right)$ denoted the multiplication operator by the function $\gamma_{\ell}^{m}$. Condition (5.54) yields

$$
E L E_{+\infty}=-(\mathbb{1}+E L E) E L^{b} E_{+\infty}
$$

or. in the coordinate notation. for $m \Xi S$,

$$
\begin{gathered}
D\left(L_{+\infty}^{m}\right)=H^{1}\left(\mathbb{R}^{d} ; \mathbb{C}\right), \\
L_{+\infty}^{m}=-\sum_{\ell=1}^{d}\left(\delta_{\ell}^{m}+\gamma_{\ell}^{m}\right)\left(L_{\ell}^{-\infty}\right)^{*} \\
=-\sum_{\ell=1}^{d}\left(\delta_{\ell}^{m}+\gamma_{\ell}^{m}\right)\left(\sum_{j=1}^{d} \sigma_{\ell j} \partial_{j}+\rho_{\ell}\right)
\end{gathered}
$$

where $\delta_{\ell}^{m n}=0$ if $\ell \neq m$ and $\delta_{\ell}^{m}=1$ if $\ell=m$.

Finally let $L_{+\infty}^{-\infty}$ be the operator

$$
\begin{gathered}
D\left(L_{+\infty}^{-\infty}\right)=H^{2}\left(\mathbb{R}^{i}: \mathbb{T}\right), \\
L_{+\infty}^{-\infty}=-\frac{1}{2} \sum_{\ell=1}^{d} L_{\ell}^{-\infty}\left(L_{\ell}^{-\infty}\right)^{*}+\frac{1}{2} \sum_{j=1}^{d}\left(\eta_{j} \partial_{j}+\partial_{j} \eta_{j}\right)
\end{gathered}
$$

Notice that $\left(L_{\ell}^{-\infty}\right)^{*}$ (resp. $\left.\left(L_{+\infty}^{-\infty}\right)^{*}\right)$ coincides with the operator $L_{\ell}$ (resp. $G)$ given by (4.10) (resp. (4.13)).

The results of Chapter 4, Section 2 allow us to prove immediately the following

Proposition 5.31 The operators $L_{+\infty}^{-\infty}, L_{\ell}^{+\infty}$ satisfy the hypothesis AA as well as the operators $\left(L_{+\infty}^{-\infty}\right)^{*},\left(L_{-\infty}^{\ell}\right)^{*}$. The associated quantum dynamical semigroups are Markov.

Proof. The operators $L_{+\infty}^{-\infty},\left(L_{+\infty}^{-\infty}\right)^{*}$ are infinitesimal generators of contraction semigroups in $h$ by Theorem A.3. Indeed these are second order differential operators with sufficiently regular coefficients.

Moreover the linear manifold $C_{c}^{\infty}\left(\mathbb{R}^{d} ; \mathcal{C}\right)$ is a core for both. Therefore the identity (3.28), which is immediately checked for $u \in C_{c}^{\infty}\left(\mathbb{R}^{d} ; \mathbb{C}\right)$ by our choice of the coefficients of the differential operators $L_{+\infty}^{-\infty}, L_{\ell}^{+\infty}$ and $\left(L_{+\infty}^{-\infty}\right)^{*},\left(L_{-\infty}^{\ell}\right)^{*}$, is fulfilled. Hence condition AA holds. 
Finally, since the coefficients of $L_{+\infty}^{-\infty},\left(L_{+\infty}^{-\infty}\right)^{*}$ are sufficiently regular (Hypothesis D) holds, we can apply Theorem 4.6 to concluded that both the quantum dynamical semigroups associated with $L_{+\infty}^{-\infty}, L_{\ell}^{+\infty}$ and with $\left(L_{+\infty}^{-\infty}\right)^{*}$, $\left(L_{-\infty}^{\ell}\right)^{*}$ are Markov.

Let $K$ and $H$ be the operators

$$
\begin{aligned}
& D(K)=H^{2}\left(\mathbb{R}^{d} ; \mathcal{C}\right), \quad K=-\frac{1}{2} \sum_{\ell=1}^{d} L_{\ell}^{-\infty}\left(L_{\ell}^{-\infty}\right)^{*}, \\
& D(H)=H^{1}\left(\mathbb{R}^{d} ; \mathscr{C}\right), \quad H=-\frac{i}{2} \sum_{j=1}^{d}\left(\eta_{j} \partial_{j}+\partial_{j} \eta_{j}\right)
\end{aligned}
$$

Notice that both $K$ and $H$ are self-adjoint. ( $K$ is negative self-adjoint). For every $n \geq 1$ let

$$
K_{n}=n^{2} R(n ; K) K R(n ; K), \quad H_{n}=i n H R(i n ; H)
$$

Consider bounded approximations of $L$

$$
\begin{aligned}
\left(L_{n}\right)_{\ell}^{m} & =L_{\ell}^{m} & & \text { if } \ell, m \in S, \\
\left(L_{n}\right)_{\ell}^{-\infty} & =n R(n ; K) L_{\ell}^{-\infty} & & \text { if } \ell \in S, \\
\left(L_{n}\right)_{+\infty}^{m} & =n L_{+\infty}^{m} R(n ; K), & & \text { if } m \in S, \\
\left(L_{n}\right)_{+\infty}^{-\infty} & =K_{n}+i H_{n} & & \text { if } m=-\infty, \ell=+\infty .
\end{aligned}
$$

Straightforward algebraic computations show that the operators $L_{n}$ satisfy identities (5.24). Therefore there exists a dual pair of unitary cocycles $V_{n}, \widetilde{V}_{n}$ such that

$$
d V_{n}(t)=V_{n}(t) d \Lambda_{L_{n}}(t), \quad d \widetilde{V}_{n}(t)=\widetilde{V}_{n}(t) d \Lambda_{\widetilde{L}_{n}}(t)
$$

The well-known properties of resolvent operators yield

$$
s-\lim _{n \rightarrow \infty} L_{n} \xi=L \xi, \quad s-\lim _{n \rightarrow \infty} L_{n}^{b} \xi=L^{b} \xi
$$

Theorem 5.32 The sequence $\left(V_{n}(t)\right)_{n \geq 1}\left(\right.$ resp. $\left.\left(\widetilde{V}_{n}(t)\right)_{n \geq 1}\right)$ converges strongly to a unitary operator $V(t)$ (resp. $\widetilde{V}(t)$ ) for every $t \geq 0$. The dual cocycles $(V(t))_{t \geq 0}\left(\right.$ resp. $\left.(\widetilde{V}(t))_{t \geq 0}\right)$ satisfy the quantum stochastic differential equations

$$
d V(t)=V(t) d \Lambda_{L}(t), \quad d \widetilde{V}(t)=\widetilde{V}(t) d \Lambda_{\widetilde{L}}(t)
$$

with initial conditions $V(0)=\mathbb{1}, \widetilde{V}(0)=\mathbb{1}$.

Proof. The identity (5.39) for the operators $L, \widetilde{L}$ is immediately checked by our choice of the coefficients of the differential operators $L_{\ell}^{m}$. Therefore there exists unique contractive solutions of the above quantum stochastic differential equations. 
Since the associated quantum dynamical semigroup are Markov by Proposition 5.31, the cocycles $(V(t))_{t \geq 0},(\widetilde{V}(t))_{t \geq 0}$ are isometric. Thus, being a pair of dual cocycles, they are unitary.

By virtue of Proposition 5.16 there exists a subsequence $\left(n_{m}\right)_{m \geq 1}$ such that

$$
V(t)=w-\lim _{m \rightarrow \infty} V_{n_{m}}(t), \quad \tilde{V}(t)=w-\lim _{m \rightarrow \infty} \tilde{V}_{n_{m}}(t) .
$$

for every $t \geq 0$.

The above limits are also strong because both the operators $V(t)$ and $\widetilde{V}(t)$ are isometries.

Finally we can remove subsequences by the same argument of the proof of Proposition 5.21 because limit cocycles are unique.

We find now, applying Theorem 5.28, the quantum stochastic differential equation satisfied by the quantum flow obtained by conjugation through the unitary cocycle $V$. In order to show that the operators ${ }^{(n)} \theta_{m}^{\ell}(f)$ with $f \in \mathcal{A}_{\infty}$ are uniformly bounded in norm we need the following easy lemma.

Lemma 5.33 For every $f \in \mathcal{A}_{\infty}$ the operators

$$
R(n ; K)[K,[K, f]] R(n ; K)
$$

are uniformly bounded in norm.

Proof. A straightforward computation shows that the double commutator $[K,[K, f]]$ is given by the multiplication operator by

$$
\frac{1}{2} \sum_{j, k=1}^{d} a_{j k}(x)\left(\partial_{j} \partial_{k} f\right)(x)+\sum_{k=1}^{d} \tilde{b}_{k}(x)\left(\partial_{k} f\right)(x)
$$

where

$$
\tilde{b}_{k}(x)=\frac{1}{2} \sum_{m=1}^{d}\left(\partial_{m} a_{m k}\right)(x)+\sum_{m=1}^{d} \rho_{m}(x) \sigma_{m k}(x) .
$$

Indeed the second order differential operator $f \rightarrow[K,[K, f]]$ must coincide with the second order differential operator given by the choice $\eta=0$ in (5.56).

The conclusion follows from the fact that the operators $R(n ; K)$ are contractions.

Proposition 5.34 Let $\mathcal{A}=C_{l}^{0}\left(\mathbb{R}^{d} ; \mathbb{R}\right)$ and let $\mathcal{A}_{\infty}$ be the algebra generated by compact support functions and constant functions and let $(V(t))_{t \geq 0}$ be the unitary cocycle satisfying the quantum stochastic differential equation (5.58). The quantum flow

$$
j_{t}(x)=V(t) x V(t)^{*}, \quad x \in \mathcal{B}(h)
$$


satisfies, for $f \in \mathcal{A}_{\infty}$, the quantum stochastic differential equation (5.43) with structure maps

$$
\begin{array}{rlrl}
\theta_{m}^{\ell}(f) & =0, & \ell, m \in S \\
\theta_{+\infty}^{m}(f) & =-\sum_{k, j=1}^{d}\left(\delta_{k}^{m}+\gamma_{k}^{m}\right) \sigma_{k j}\left(\partial_{j} f\right), & & m \in S \\
\theta_{\ell}^{-\infty}(f) & =-\sum_{k, j=1}^{d}\left(\delta_{k}^{\ell}+\gamma_{k}^{\ell}\right) \sigma_{k j}\left(\partial_{j} f\right), & & \ell \in S \\
\theta_{+\infty}^{-\infty}(f) & =\frac{1}{2} a_{j k}\left(\partial_{j} \partial_{k} f\right)+b_{j}\left(\partial_{j} f\right) &
\end{array}
$$

where $\left(b_{j}\right)_{1 \leq j \leq d}$ is given by (5.56).

Proof. Clearly $\mathcal{A}_{\infty}$ is dense in $\mathcal{A}$ for the uniform norm.

We check the hypotheses 1 and 2 of Theorem 5.28. For every $f \in \mathcal{A}_{\infty}, n \geq 1$, $\ell, m \in\{1, \ldots, d\}$, by the basic rules of quantum stochastic calculus, we have

$$
\begin{aligned}
{ }^{(n)} \theta_{m}^{\ell}(f)= & \theta_{m}^{\ell}(f)=0 \\
{ }^{(n)} \theta_{+\infty}^{m}(f)= & n L_{+\infty}^{m} R(n ; K) f+n f\left(L_{n}^{-\infty}\right)^{*} R(n ; K) \\
& +n \sum_{k=1}^{d} \gamma_{k}^{m} f\left(L_{k}^{-\infty}\right)^{*} R(n ; K) \\
{ }^{(n)} \theta_{\ell}^{-\infty}(f)= & n R(n ; K) L_{\ell}^{-\infty} f+n f R(n ; K)\left(L_{+\infty}^{\ell}\right)^{*} \\
& +n R(n ; K) \sum_{k=1}^{d} L_{k}^{-\infty} f \gamma_{k}^{\ell} \\
{ }^{(n)} \theta_{+\infty}^{-\infty}(f)= & i\left[H_{n}, f\right]+K_{n} f+f K_{n} \\
& +\sum_{j=1}^{d} n R(n ; K) L_{\ell}^{-\infty} f n\left(L_{\ell}^{-\infty}\right)^{*} R(n ; K)
\end{aligned}
$$

Therefore hypothesis 1 of Theorem 5.28 follows from the well-known properties of resolvent operators.

In order to check the hypothesis 2 notice first that structure maps ${ }^{(n)} \theta_{m}^{\ell}$ with $\ell, m \in\{1, \ldots, d\}$ are obviously uniformly bounded in $n$. Then, due to the structure relation,

$$
{ }^{(n)} \theta_{+\infty}^{-\infty}(y x)=y^{(n)} \theta_{+\infty}^{-\infty}(x)+{ }^{(n)} \theta_{+\infty}^{-\infty}(y) x+\sum_{\ell=1}^{d}{ }^{(n)} \theta_{\ell}^{-\infty}(y)^{(n)} \theta_{+\infty}^{\ell}(x)
$$

it suffices to prove that, for every $f \in \mathcal{A}_{\infty}$, the operators ${ }^{(n)} \theta_{+\infty}^{-\infty}(f)$ are uniformly bounded in $n$. 
A direct computation from (5.64) yields

$$
\begin{aligned}
{ }^{(n)} \theta_{+\infty}^{-\infty}(f)= & i\left[H_{n}, f\right]+K_{n} f+f K_{n} \\
& +\sum_{j=1}^{d} n R(n ; K) L_{\ell}^{-\infty} f n\left(L_{\ell}^{-\infty}\right)^{*} R(n ; K) \\
= & i\left[H_{n}, f\right]+n R(n ; K) \theta_{+\infty}^{-\infty}(f) n R(n ; K) \\
& +n^{2} R(n ; K)[R(n ; K), f]+n^{2}[f, R(n ; K)] R(n ; K)
\end{aligned}
$$

The second term is obviously uniformly bounded. The first one can be written in the form

$$
\begin{aligned}
i\left[H_{n}, f\right] & =n H R(i n ; H) f-n f H R(i n ; H) \\
& =n H[f, R(i n ; H)]+n[f, H] R(i n ; H) \\
& =H R(i n ; H)[H, f](n R(i n ; H))+[f, H](n R(i n ; H)) .
\end{aligned}
$$

Since $[f, H]$ is a bounded multiplication operator, the operators $\left[H_{n}, f\right]$ are uniformly bounded.

The sum of the third and fourth torm can be written in the form

$$
\begin{aligned}
& n^{2}(R(n ; K))^{2}[f, n-K] R(n ; K)+n^{2} R(n ; K)[n \cdots K, f](R(n ; K))^{2} \\
= & (n R(n ; K))(R(n ; K)[K, f]-[K, f] R(n ; K))(n R(n ; K)) \\
= & (n R(n ; K))[R(n ; K),[K, f]](n R(n ; K)) \\
= & (n R(n ; K))\{R(n ; K)[K,[K, f]] R(n ; K)\}(n R(n ; K)) .
\end{aligned}
$$

Therefore it is uniformly bounded in norm by Lemma 5.33 .

We can now prove the main result of this section.

Theorem 5.35 The restriction of the quantum flow (5.60) to the algebra $\mathcal{A}$ coincides with the flow of a classical diffusion with infinitesimal generator $\theta_{+\infty}^{-\infty}$.

Proof. The algebra $\mathcal{A}_{\infty}$ is a core for $\theta_{+\infty}^{-\infty}$ by Theorem A.1. Moreover the structure maps $\theta_{m}^{\ell}(\ell, m \in\{1, \ldots, d,+\infty,-\infty\})$ of the quanturn flow (5.60) map $\mathcal{A}_{\infty}$ into $\mathcal{A}$. Therefore, by virtue of Theorem 5.30 , the algebra $\mathcal{A}$ is invariant under the homomorphisms $j_{t}$ and the restriction to $\mathcal{A}$ of the quantum flow (5.60) is commutative.

Proposition 5.34 implies that it satisfies the quantum stochastic differential equation

$$
\left.\left.d j_{t}(f)=\sum_{\ell=1}^{d} j_{t}\left(\theta_{\ell}^{-\infty}(f)\right)\right)\left(d \Lambda_{-\infty}^{\ell}(t)+d \Lambda_{\ell}^{-\infty}(t)\right)+j_{t}\left(\theta_{+\infty}^{-\infty}(f)\right)\right) d t
$$

with $j_{0}(f)=f$. Interpreting $\left(\Lambda_{-\infty}^{\ell}(t)+\Lambda_{\ell}^{-\infty}(t)\right)_{t \geq 0}$ as an operator version of classical Brownian motion via the Segal isometry it turns out that the above equation coincides with the stochastic differential equation for the flow of a diffusion process with infinitesimal generator $\theta_{+\infty}^{-\infty}$. 


\subsection{Conclusion}

The boson Fock quantum stochastic calculus developed by Hudson and Parthasarathy [58] allows to construct several quantum flows by conjugation with unitary cocycles extending the flow of a classical Markov process to the algebra of all bounded operators on a Hilbert space which is often an $L^{2}$ space of the state space. Since q.s.d.e. are driven by brownian motions and Poisson processes which are mutually non-commuting, in this way we can realise a classical Markov process as a non-commutative functional of brownian motions and Poisson processes. Moreover the quantum flow enjoys the quantum Markov property in the sense of Accardi [1] and satisfies a quantum stochastic differential equation.

A class of quantum flows which are not necessarily of the form

$$
j_{t}(x)=V(t) x V(t)^{*}
$$

has been studied by Evans and Hudson in [36]. A detailed account on this subject for classical Markov processes with bounded generator can be found in the recent books by Meyer [68] and Parthasarathy [74].

The generalisation to unbounded generators is necessary to construct a noncommutative extension of flows of most interesting classical Markov processes. Here we described the general scheme (outlined in [41]) for constructing such extension. The main steps are the following:

1. from the infinitesimal generator of a classical process find a possible choice of the structure maps of the quantum stochastic differential equation satisfied by the flow $j$ and the coefficients of the quantum stochastic differential equation satisfied by the cocycle $V$,

2. show the existence of $V$ by an approximation procedure,

3. show that $V$ is unitary,

4. study a "regularity" property of a sequence $\left\{j^{(n)}\right\}_{n \geq 1}$ of approximating quantum flows with bounded structure maps,

5. show that the homomorphisms $\left\{j_{t}\right\}_{t \geq 0}$ are also homomorphism of a smaller commutative algebra.

This scheme has been successfully followed here to construct quantum flows extending the classical flow of multidimensional diffusion processes with "regular" covariance and drift. The same can be done for discrete state quantum Markov chains (see [70]) and other classical stochastic processes as for example Bessel processes (see [71] and also [17] with a different approach for integer dimension index).

The analytical methods described here can be used also to construct quantum diffusions on manifold studied by Applebaum in [11] and Sauvageot in [80] mainly from the algebraic point of view.

The success of this scheme depends essentially on the form of infinitesimal generator. Some techniques remind constructions of classical Markov processes 
done in [33]. The most difficult problem arises when it turns out that the cocycle $V$ is not isometric (see, for example, [19], [38] and [44]). This always occurs when the classical process has some "singular state", for example a boundary point that can be reached in finite time, so that the infinitesimal generator depends on boundary conditions. In this case the minimal quantum dynamical semigroup arising from the representation in Lindblad form of the infinitesimal generator might not be identity preserving. Therefore we expect that the cocycle $V$ satisfies a very singular quantum stochastic differential equation.

The attempts to study directly the quantum stochastic differential equation

$$
d j_{t}(x)=\sum_{\ell, m \in \bar{S}} j_{t}\left(\theta_{m}^{i}(x)\right) d \Lambda_{\ell}^{m}(t)
$$

have been successful only when the structure maps are bounded [34] or satisfy and analyticity condition which is very hard to check in practice [48].

A result of Accardi and Mohari [9] states that, given a family of homomorphisms $\left(j_{t}\right)_{t \geq 0}$, if the domain of the infinitesimal generator of the associated quantum Markov semigroup is an algebra, then the quantum flow must satisfy an equation of the form (5.65). However there are no known results (besides those in the classical commutative cases and [48] in the noncommutative one) allowing to prove that given unbounded structure maps satisfying the structure relations the maps $\left(j_{t}\right)_{t \geq 0}$ solving (5.65) are homomorphisms.

The direct study of the equation for $\left(j_{t}\right)_{t \geq 0}$ however scems important because, even in the simplest cases, it might not be possible to find a suitable choice of the coefficients of equation for $V$. In fact consider, for example, the classical Markov process on $\mathbb{R}$ with infinitesimal generator

$$
(A f)(x)=f(0)-f(x) .
$$

The heuristic discussions in Chapter 4 and 5 lead us to the choice

$$
-\left(\theta_{0}^{1}(f)\right)(x)=-\left(\theta_{1}^{0}(f)\right)(x)=\left(\theta_{1}^{1}(f)\right)(x)=f(0)-f(x)
$$

and it is not possible to find a unitary operator on $L^{2}(\mathbb{R})$ such that $\left(S f S^{*}\right)(x)=$ $f(0)$. 